\title{
Reaction Mechanism and Deactivation Modes of Heterogeneous Catalytic Systems
}

\author{
K. KUMBILIEVA ${ }^{1}$, L. PETROV ${ }^{2, *}$, Y. ALHAMED ${ }^{2}$, A. ALZAHRANI ${ }^{2}$ \\ ${ }^{1}$ Institute of Catalysis, Bulgarian Academy of Sciences, Sofia, Bulgaria \\ ${ }^{2}$ SABIC Chair of Catalysis, King Abdulaziz University, Jeddah, Saudi Arabia
}

\begin{abstract}
Solving the problem of catalyst deactivation is essential in process design. To do this, various aspects of the kinetics of processes with catalyst deactivation, and their different mechanisms, are discussed. Catalyst deactivation often cannot be avoided, but more knowledge on its mechanism can help to find kinetic means to reduce its harmful consequences. When deactivation is caused by coke, the generation of coke precursors is the determining step in the deactivation kinetics. Different types of deactivation were distinguished that lead to different evolution of the process. The phenomenon of non-uniform coking can be linked to catalyst surface non-uniformity. For the class of catalysts with more than one type of active sites, an explanation was suggested for the observed trends in the deactivation modes. For catalytic processes using catalyst particles of industrial size, the influence of intraparticle diffusion resistance is important. The analysis showed that for a number of processes, the decrease of the reaction rate due to deactivation is less under diffusion control. For certain reaction mechanisms, there exist operation conditions where the rate of the process under diffusion control exceeds the rate in the kinetic control regime. A significant problem is the change of selectivity in the course of catalyst deactivation. The selectivity may either decrease or increase, and depends on the reaction mechanism during deactivation. The changes are larger when there is no diffusion resistance. The intentional poisoning of catalysts and its influence on catalyst activity and selectivity for the process of ethylene oxide production was discussed.
\end{abstract}

Key words: catalyst activity; catalyst selectivity; catalyst deactivation; active site; diffusion regime

CLC number: O643

Document code: A

A major problem in the use of heterogeneous catalysts is the loss of catalyst activity and/or selectivity in the course of reaction. The changes brought about by catalyst deactivation play a significant, although disadvantageous, role in a large number of important industrial processes. There are no scientific or practical approaches that can entirely eliminate catalyst deactivation. Researcher efforts are directed at finding ways to reduce the harmful consequences of the deterioration of catalyst activity and selectivity. In the past three decades, there was much motivation for understanding catalyst decay. A series of thorough studies, esp. [1-14], addressed a variety of deactivation phenomena. It is of academic and industrial interest to have more detailed knowledge on the kinetics of processes accompanied by catalyst deactivation, and its connection with the reaction mechanism.

Different approaches have been developed to study various aspects of the effects caused by the change in catalyst activity. Two strategies can be distinguished.

(i) A wide class of works correlate the evolution of catalyst activity using empirical data from various conditions. This strategy was stimulated by the recent development of advanced precise techniques and methods that record the responses of the catalytic system to changes of the input characteristics to construct a picture of the system behavior. The majority of deactivation models derived by this approach do not consider the mechanistic features of the catalytic process. When based on good physicochemical observations, this approach is important for its collecting of reliable data that is informative of the evolution of the particular catalytic systems under investigation, but the conclusions drawn often cannot be extrapolated, and can be applied only for the reactor under study.

(ii) The construction of models that do not depend on the parameters of a particular reactor, which needs a strategy to correlate the observed deactivation with the mechanism. In this approach, the developing of more efficient catalysts uses the deeper insight on the kinetics and mechanism that lead to the decrease of catalytic activity [1-14].

A widely recognized classification suggested by Hughes [1] provisionally divides deactivation according to the reasons causing the loss of catalyst activity: (i) poisoning of catalysts by impurities in the reaction mixture; (ii) sintering of the catalyst; and (iii) changes in the catalyst activity provoked by reaction routes that are a constituent part of the reaction mechanism. Bartholomew [2] also defined some

Received 28 September 2010. Accepted 8 November 2010.

*Corresponding author. Tel: +966-507589382; Fax: +966-2-6952257; E-mail: lpetrov@kau.edu.sa

English edition available online at ScienceDirect (http://www.sciencedirect.com/science/journal/18722067). 
other distinct mechanisms of deactivation: (iv) vapor-solid and solid-solid reactions with the catalyst phase to produce inactive or volatile compounds; and (v) attrition/crushing.

The first two types of deactivation can be studied separately from the mechanism of the reactions. The consequences of impurity poisoning can be reduced by the purification of the reaction mixture, which makes the problem technological rather than kinetic. Detailed analysis of catalyst deactivation by poisoning can be found in Refs. $[1-4,6,9,12]$.

In some cases, however, intentional poisoning is used for improving catalyst selectivity, which also leads to slight decrease of catalytic activity. The addition of dichoroethane as selectivity promotor is used in the process of ethylene oxide production. A detailed kinetic study of the process of ethylene oxidation to ethylene oxide in the presence of dichoroethane has been presented in Refs. [15-19].

Sintering (or thermal deactivation) generally takes place at high reaction temperatures (above $500{ }^{\circ} \mathrm{C}$ ), and results from the loss of either catalyst surface area due to crystalline growth, or of support area due to support collapse. Insofar as the temperature is the most important factor responsible for thermal deactivation, the main efforts for its prevention are oriented on establishing reasonable optimal temperature regime for the process in order to ensure a satisfactory reaction rate, and yet avert drastic deterioration of the catalyst structure. Many problems associated with vapor-solid and solid-solid state reactions resulting in deactivation are still poorly understood. The problems related to loss of catalyst activity due to attrition are more subject to mechanical solutions.

A special class of problems can be associated with deactivation originating from interactions with species participating in the reaction mechanism. Generally, these effects can be specified as follows:

(i) Strong (sometimes irreversible) adsorption of initial reactants, or final products, or intermediate species that occur in the elementary steps in the reaction mechanism. Some authors define these effects as "self-poisoning". It is worth noting the essential difference of this type of deactivation effects from the effects originating from impurity poisoning. Purification can provide protection from impurity poisoning, but cannot prevent self-poisoning because the species responsible for the latter are participants in the process.

(ii) Interactions of reactants with some catalyst centers, resulting in changes of valency state, formation of undesired chemicals, etc.

(iii) Penetration through the surface layer or induced diffusion of the components of the catalyst lattice towards the catalyst surface.

(iv) Blocking of active catalyst sites by coke deposits.
Coke formation is considered the most common reason for catalyst deactivation. It is a widely held assumption that the generation of coke precursors is the reason for coke formation. For elucidating the correlation between the reaction mechanism and catalyst deactivation, it is of interest to focus on those cases where the formation of coke precursors is due to interactions in the reaction mechanism. In this view, the scheme suggested by Wolf and Petersen [20,21] is of importance for understanding the principles of the process. According to this scheme, the mechanism of coking is specified as parallel in case the coke precursors originate from species or surface intermediates formed before the slow (rate determining) step of the main reaction. The series (consecutive) mechanism of coke formation is defined as that when surface intermediates generated after the slow step are transformed into coke precursors. There may also be interactions of intermediates of different types, which corresponds to a combined scheme of coking.

Catalyst deactivation obeys different kinetics according to the mechanism of the blockage of active sites. Accordingly, the changes of catalyst performance due to catalyst deactivation can follow different trends, e.g. sharp drop in catalytic activity at the beginning of the process or a smooth or stepwise decrease of catalytic activity.

In the kinetic description of the influence of catalyst deactivation on reaction performance, two common approaches are usually applied, namely, "separable deactivation kinetics" [22,23] and "unseparable deactivation kinetics" [24].

In the "separable deactivation kinetics" approach, the rate of the entire catalytic process is expressed in the form of a product of the function of the initial reaction rate of the fresh catalyst and a function characterizing the change of the catalyst activity by deactivation.

The "non-separable deactivation" approach is based on the principle that there should be a correspondence between the kinetics of the main reaction, the kinetics of generation of blocking agents (coke in particular) and the kinetics of deactivation. Such an approach is more rigorous as it takes into account the correlation of deactivation with the mechanism of the overall process. On the other hand, the "separable deactivation" approximation is often more convenient for kinetic simulations. We discuss below the application of both approaches.

\section{Mechanistic considerations}

Models that use the approximation of separable deactivation kinetics $[22,23]$ express the reaction rate as the product of the initial reaction rate $r^{0}$ and a function $a(u)$ that is related to the current catalytic activity:

$$
r(u)=r^{0} \cdot a(u)
$$


where $u$ may be time on stream $t$, or degree of conversion $x$, amount of coke $C$, etc.

It is important to distinguish clearly the function of catalyst activity (which will be denoted as $a\left(u_{j}\right)$ ), and the deactivation function (denoted as $\phi\left(u_{j}\right)$ ). The $u_{j}$ arguments of these functions may be various factors which play key roles in deactivation phenomena. In the simplest case, these functions are considered as time dependent, $(u \equiv t)$, and the analysis of the behavior of the reaction system has to be linked to the analysis of the $a(t)$ and $\phi(t)$ functions.

Usually, the activity is defined as the ratio of the current reaction rate $r(t)$ and the reaction rate $r^{0}$ on the fresh catalyst:

$$
a(t)=\frac{r(t)}{r^{0}}
$$

At the beginning of the process, $r(t)_{t \rightarrow 0} \equiv r^{0}$, and $a(t)_{t \rightarrow 0}=$ 1 . When the catalyst deactivation begins, the activity function $a(t)$ will decrease with time on stream. The rate of the decrease is characterized by the deactivation function $\phi(u)$ :

$$
\phi(u)=-\frac{\mathrm{d}}{\mathrm{d} u} a(u)=-\frac{\mathrm{d}}{\mathrm{d} u}\left(\frac{r(u)}{r^{0}}\right)=-\frac{1}{r^{0}} \frac{\mathrm{d} r}{\mathrm{~d} u}
$$

Different expressions can be derived to describe the deactivation function $\phi(u)$, depending on the deactivation mechanism. With the approximation $u \equiv t$, Eq. (3) takes the form:

$$
\phi(t)=-\frac{\mathrm{d}}{\mathrm{d} t} a(t)=-\frac{\mathrm{d}}{\mathrm{d} t}\left(\frac{r(t)}{r^{0}}\right)=-\frac{1}{r^{0}} \frac{\mathrm{d} r}{\mathrm{~d} t}
$$

The activity of the catalyst at time $\left(t_{\mathrm{a}}\right)$ can be determined from

$$
(a(t))_{t=t_{\mathrm{a}}}=1-\int_{0}^{t=t_{\mathrm{a}}} \phi(t) \mathrm{d} t
$$

The reaction rate within the framework of separable deactivation kinetics can be defined by

$$
(r(t))_{t=t_{\mathrm{a}}}=r^{0} \cdot\left(1-\int_{0}^{t=t_{\mathrm{a}}} \phi(t) \mathrm{d} t\right)
$$

This approximation does not require a correspondence between the apparent rate of the process and the mechanism of deactivation. Nonetheless, observations and models concerning particular processes differ by the types of "deactivation functions" that describe different deactivation mechanisms.

When the major reason for deactivation is coke formation from feed species or products, it is reasonable to consider the degree of conversion $(x)$ as an argument for the deactivation function. Thus, some of the most common mechanistic types of deactivation have been described in terms of the degree of conversion as follows.

Deactivation through dissociative adsorption of feed spe- cies:

$$
\phi(x)=-\beta \sqrt{(1-x)}
$$

Deactivation through dissociative adsorption of product:

$$
\phi(x)=-\beta \sqrt{x}
$$

Deactivation through one-center adsorption of feed species:

$$
\phi(x)=-\beta(1-x)
$$

Deactivation through one-center adsorption of product:

$$
\phi(x)=-\beta x
$$

Deactivation through two-center adsorption of feed:

$$
\phi(x)=-\beta(1-x)^{2}
$$

Deactivation through two-center adsorption of product:

$$
\phi(x)=-\beta x^{2}
$$

Deactivation by product adsorption on exponentially inhomogeneous surfaces:

$$
\phi(x)=\exp (-\beta x)
$$

It is reasonable, since the main reason for deactivation is the blockage of active sites, to describe the deactivation function in terms of the surface concentration of blocked active sites $\left(\theta_{\mathrm{c}}\right)$. In the most common cases,

$$
\phi\left(\theta_{\mathrm{c}}\right)=\exp \left(\varepsilon \theta_{\mathrm{c}}\right)
$$

or

$\phi\left(\theta_{\mathrm{c}}\right)=\xi\left(1-\theta_{\mathrm{c}}\right)^{n}(n=0.5,1$, or 2 , depending on the deactivation mechanism)

For $n=1$, the activity drop versus $\theta_{\mathrm{c}}$ is linear, abrupt or steep, depending on the strength of the sites eliminated. For $n=2$ and not very high values of the $\xi$ parameter, the deactivation may manifest a stepwise character - being initially negligible and becoming apparent only after attaining a critical value $\theta_{\mathrm{c}}^{*}$ of the blocked fraction of active sites.

The non-separable deactivation approach considers the correlation of deactivation kinetics with the mechanism of the main process, and the origin of sources for coke precursors.

The general scheme of reaction occurring on single catalyst sites may be presented as

$$
\mathrm{A}+\mathrm{Z} \leftrightarrow[\mathrm{AZ}]_{1} \leftrightarrow \cdots \leftrightarrow[\mathrm{AZ}]_{j} \underset{[\mathrm{BZ}]_{m} \leftrightarrow \mathrm{B}+\mathrm{Z}}{\stackrel{k_{\text {slow }}}{\longrightarrow}}[\mathrm{BZ}]_{j+1} \leftrightarrow \cdots \leftrightarrow
$$

where $\mathrm{A}$ and $\mathrm{B}$ denote, respectively, the key initial reactant and the product, $Z$ is an active site on the catalyst surface, $[\mathrm{AZ}]_{1},[\mathrm{AZ}]_{j},[\mathrm{BZ}]_{j+1},[\mathrm{BZ}]_{m}$, stand for surface intermediates formed, respectively, before and after the slow step $[\mathrm{AZ}]_{j}$ $\stackrel{k_{\text {slow }}}{\longrightarrow}[\mathrm{BZ}]_{j+1}$. In these terms, and denoting by (PZ) the coke precursor, the schemes of parallel and series coke formation specified by Wolf and Petersen may be presented as

$$
\begin{aligned}
{[\mathrm{AZ}]_{m} } & \rightarrow(\mathrm{PZ}) \rightarrow \text { coke } \quad(\text { parallel }) \\
{[\mathrm{BZ}]_{m} } & \rightarrow(\mathrm{PZ}) \rightarrow \text { coke } \quad \text { (series) }
\end{aligned}
$$

where (PZ) is the coke precursor. Usually coke formation is initialized by the adsorption of gas feed species or product molecules on catalyst sites. The intermediates formed may be further transformed into coke precursors, and this is often 
coupled with interactions with molecules from the gas phase. In view of this, the deactivation function should include the surface concentrations of intermediates as well as partial pressures of the gas reactants responsible.

Following these considerations, we have extended $[25,26]$ the classification proposed by Wolf and Petersen to include the following typical mechanisms for the generation of coke precursors.

Type 1: The coke precursor is generated through interaction of feed species (A) with some intermediate $\left([\mathrm{AZ}]_{j}\right)$ formed before the slow step of the main reaction

$$
n \mathrm{~A}+[\mathrm{AZ}]_{j} \stackrel{\text { slow }}{\longrightarrow}(\text { PrecZ }) \longrightarrow \text { coke }
$$

The coke formation rate is

$$
r_{\mathrm{C}}=k_{\mathrm{d}} P_{\mathrm{A}}^{n} \theta_{\mathrm{A}_{j}}
$$

Type 2: The coke precursor is generated through interaction of product molecules (B) with some intermediate $\left([\mathrm{AZ}]_{j}\right)$ formed before the slow step of mechanism

$$
n \mathrm{~B}+[\mathrm{AZ}]_{j} \stackrel{\text { slow }}{\longrightarrow}(\text { PrecZ }) \longrightarrow \text { coke }
$$

The coke formation rate is

$$
r_{\mathrm{C}}=k_{\mathrm{d}} P_{\mathrm{B}}^{n} \theta_{\mathrm{A}_{j}}
$$

Type 3: The coke precursor is generated through interaction of feed species molecules with some intermediate $\left([\mathrm{BZ}]_{j}\right)$ formed after the slow step after the mechanism

$$
n \mathrm{~A}+[\mathrm{BZ}]_{j} \stackrel{\text { slow }}{\longrightarrow}(\text { PrecZ }) \longrightarrow \text { coke }
$$

The coke formation rate is

$$
r_{\mathrm{C}}=k_{\mathrm{d}} P_{\mathrm{A}}^{n} \theta_{\mathrm{B}_{j}}
$$

Type 4: The coke precursor is generated through interaction of product molecules with some of the intermediates $\left([\mathrm{BZ}]_{j}\right)$ formed after the slow step after the mechanism

$$
n \mathrm{~B}+[\mathrm{BZ}]_{j} \stackrel{\text { slow }}{\longrightarrow}(\text { PrecZ }) \longrightarrow \text { coke }
$$

The coke formation rate is

$$
r_{\mathrm{C}}=k_{\mathrm{d}} P_{\mathrm{B}}^{n} \theta_{\mathrm{B}_{j}}
$$

$P_{\mathrm{A}}$ and $P_{\mathrm{B}}$ denote the partial pressures of feed and product species, $\theta$ is the fraction of free active sites, the $\theta_{j}$ variables stand for the fractions of the surface occupied by species of surface intermediates as follows

$$
\begin{gathered}
\theta=\frac{[\mathrm{Z}]}{[\mathrm{Z}]+\sum_{1}^{j}[\mathrm{AZ}]_{i}+\sum_{j+1}^{m}[\mathrm{BZ}]_{i}} \\
\theta_{\mathrm{A}_{j}}=\frac{[\mathrm{AZ}]_{i}}{[\mathrm{Z}]+\sum_{1}^{j}[\mathrm{AZ}]_{i}+\sum_{j+1}^{m}[\mathrm{BZ}]_{i}} \\
\theta_{\mathrm{B}_{j}}=\frac{[\mathrm{BZ}]_{i}}{[\mathrm{Z}]+\sum_{1}^{j}[\mathrm{AZ}]_{i}+\sum_{j+1}^{m}[\mathrm{BZ}]_{i}}
\end{gathered}
$$

Usually the limiting step for the coking process is the formation of coke precursors. The various mechanisms for the formation of coke precursors include interactions between different surface intermediates with either initial reactants or products from the gas phase. When the coke precursors are generated after Schemes (17) or (18), the kinetics of coke formation depends on the surface concentrations of the intermediates. With some of the Schemes (21)-(25), the kinetic description of deactivation is a function of both surface concentrations and partial pressures of the participants involved. Following this approach, different types of deactivation schemes are distinguished that lead to different evolution of the coking process.

The deactivation kinetics is a function of the surface concentrations of the intermediates and the partial pressures of the gas phase reactants involved

$$
\phi=\phi\left(P_{j}(x), \theta_{i}, \theta_{s}(t)\right)
$$

Various deactivation models were derived on the basis of specific mechanisms. The deactivation kinetics was related to the peculiarities of the generation of the deactivating agents. The kinetic models corresponding to these simplest mechanisms of coke formation are shown in Table 1. The symbol $\sigma$ stands for the fraction of unblocked active sites. The expressions given in Table 1 for the rates of the main reaction and for those of coke formation proceeding by different mechanisms correspond to models of non-separable deactivation kinetics. The rates of coke formation have different functional dependencies on the conversion for each particular case.

Figure 1 shows the deactivation versus conversion curves, which characterize how the coke formation rates depend on conversion for the various mechanisms. As can be seen from these curves, the trend in the change of coke formation rate with increasing conversion can indicate the type of coke formation mechanism.

\section{Deactivation kinetics and intra-particle diffusion effects}

Mass and heat transfer in catalyst particles and catalytic reactors have a very strong influence on catalytic reactions. Different aspects of the problem were highlighted in a series of interesting works, among which we shall mention $[1,4,7$, 13,27-33]. When heterogeneous catalytic processes are carried out on catalyst particles of industrial size, an important problem is to avoid the influence of intraparticle diffusion resistance on the reaction rate since in the internal diffusion limited regime, the reaction rate is substantially reduced. However, when catalyst deactivation takes place during the catalytic process in the internal diffusion limited regime, its influence on catalyst performance is not known. 
Table 1 Schemes and simulation kinetic descriptions of the simplest mechanism patterns of coke formation accompanying reactions of the type A + $\mathrm{Z} \rightarrow \mathrm{B}+\mathrm{Z}[26]$

\begin{tabular}{|c|c|c|c|}
\hline Scheme & Mechanism pattern & $\begin{array}{l}\text { Conversion-dependent } \\
\text { term }\left(F_{\mathrm{n}}\right) \text { of the main } \\
\text { reaction rate }\end{array}$ & $\begin{array}{l}\text { Conversion-dependent term } \\
\left(F_{\mathrm{m}}\right) \text { of the rate of formation of } \\
\text { coke precursors }\end{array}$ \\
\hline Parallel & $\begin{array}{c}\mathrm{A}+\mathrm{Z} \rightarrow[\mathrm{AZ}] \cdots \stackrel{\text { slow }}{\longrightarrow}[\mathrm{BZ}] \cdots \rightarrow[\mathrm{MZ}] \rightarrow \mathrm{M}+\mathrm{Z} \\
{[\mathrm{AZ}] \stackrel{\text { slow }}{\longrightarrow}(\mathrm{PZ}) \rightarrow \cdots \rightarrow \text { coke }}\end{array}$ & $\frac{k(1-x) \sigma}{1+K_{\mathrm{s}} P_{0}(1-x)}$ & $\frac{k_{\mathrm{d}} P_{0}(1-x) \sigma}{1+K_{\mathrm{A}} P_{0}(1-x)+K_{\mathrm{B}} P_{0} x}$ \\
\hline Series & $\begin{aligned} \mathrm{A}+\mathrm{Z} \rightarrow & {[\mathrm{AZ}] \cdots \stackrel{\text { slow }}{\longrightarrow}[\mathrm{BZ}] \cdots \rightarrow[\mathrm{MZ}] \rightarrow \mathrm{M}+\mathrm{Z} } \\
& \stackrel{\mathrm{BZ}]}{\mathrm{\text {slow }}} \longrightarrow(\mathrm{PZ}) \rightarrow \cdots \rightarrow \text { coke }\end{aligned}$ & $\frac{k(1-x) \sigma}{1+K_{\mathrm{s}} P_{0} x}$ & $\frac{k_{\mathrm{d}} P_{0} x \cdot \sigma}{1+K_{\mathrm{A}} P_{0}(1-x)+K_{\mathrm{B}} P_{0} x}$ \\
\hline Parallel-parallel & 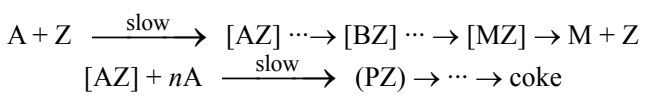 & $\frac{k(1-x) \sigma}{1+K_{\mathrm{s}} P_{0}(1-x)}$ & $\frac{k_{\mathrm{d}}\left(P_{0}\right)^{n+1}(1-x)^{n+1} \sigma}{1+K_{\mathrm{A}} P_{0}(1-x)}$ \\
\hline Parallel-consecutive & 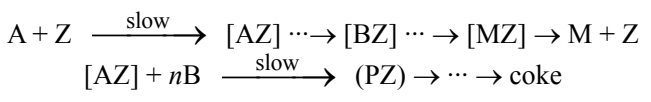 & $\frac{k(1-x)\left(1-C_{1} P_{0}\right) \sigma}{1+K_{\mathrm{s}}(1-x)}$ & $\frac{k_{\mathrm{d}}\left(P_{0}\right)^{n+1}(1-x) \cdot x^{n} \sigma}{1+K_{\mathrm{B}} P_{0} x}$ \\
\hline Series-parallel & $\begin{array}{c}\mathrm{A}+\mathrm{Z} \rightarrow[\mathrm{AZ}] \cdots \stackrel{\text { slow }}{\longrightarrow}[\mathrm{BZ}] \cdots \rightarrow[\mathrm{MZ}] \rightarrow \mathrm{M}+\mathrm{Z} \\
\quad[\mathrm{BZ}]+n \mathrm{Alow} \\
\stackrel{\text { slo }}{\longrightarrow}(\mathrm{PZ}) \rightarrow \cdots \rightarrow \text { coke }\end{array}$ & $\frac{k(1-x) \sigma}{1+K_{\mathrm{s}}(1-x)}$ & $\frac{k_{\mathrm{d}}\left(P_{0}\right)^{n+1} x \cdot(1-x)^{n} \sigma}{1+K_{\mathrm{A}} P_{0}(1-x)}$ \\
\hline Series-consecutive & $\begin{array}{c}\mathrm{A}+\mathrm{Z} \rightarrow[\mathrm{AZ}] \cdots \stackrel{\text { slow }}{\longrightarrow}[\mathrm{BZ}] \cdots \rightarrow[\mathrm{MZ}] \rightarrow \mathrm{M}+\mathrm{Z} \\
\quad[\mathrm{BZ}]+n \mathrm{~B} \stackrel{\text { slow }}{\longrightarrow}(\mathrm{PZ}) \rightarrow \cdots \rightarrow \text { coke }\end{array}$ & $\frac{k(1-x)\left(1-C_{2} P_{0}\right) \sigma}{1+K_{\mathrm{s}}(1-x)}$ & $\frac{k_{\mathrm{d}}\left(P_{0}\right)^{n+1} x^{n+1} \sigma}{1+K_{\mathrm{B}} P_{0} x}$ \\
\hline
\end{tabular}

Actually, intraparticle diffusion effects can substantially modify the catalyst deactivation dependencies. Below we discuss this issue from a theoretical point of view and will show how this can have practical applications.

\subsection{Model considerations}

Within the framework of separable deactivation kinetics,

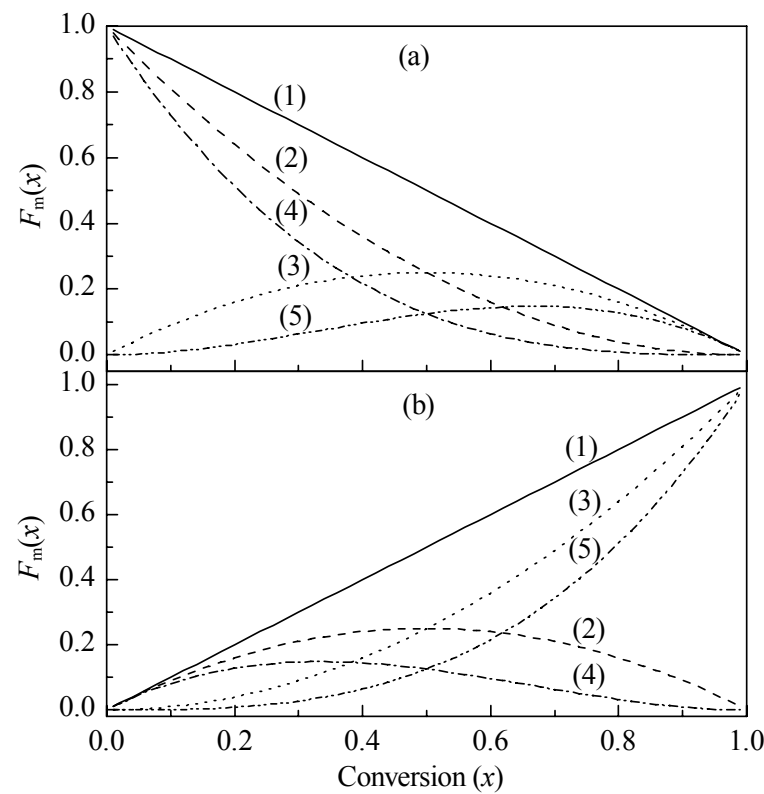

Fig. 1. Deactivation-conversion curves for various mechanisms of coke formation. (a): (1) Parallel; (2) Parallel-parallel $(n=1)$; (3) Parallel-consecutive $(n=1)$; (4) Parallel-parallel $(n=2)$; (5) Parallel-consecutive $(n=2)$. (b): (1) Series; (2) Series-parallel $(n=1)$; (3) Series-consecutive $(n=1)$; (4) Series-parallel $(n=4)$; (5) Series-consecutive $(n=2)$. the reaction rate $r_{\mathrm{k}}(x, t)$ in the kinetic region is given by the solution of the set of equations (31) and (32):

$$
\begin{aligned}
& r_{\mathrm{k}}(x, t)=k_{\mathrm{k}} r_{\mathrm{k}}^{0}\left(P_{0}, x\right) \cdot a_{\mathrm{k}}(x, t) \\
& \left(a_{\mathrm{k}}\right)_{t=t_{\mathrm{m}}}=1-\int_{0}^{t=t_{\mathrm{m}}} \phi_{\mathrm{k}}\left(P_{0}, x\right) \mathrm{d} t
\end{aligned}
$$

In the case that the reaction system is limited by intraparticle resistance, the reaction rate $r_{\mathrm{d}}(t, x)$ is given by the solution of the set of equations (33) and (34):

$$
\begin{gathered}
r_{\mathrm{d}}(x, t)=k_{\mathrm{d}} \cdot r_{\mathrm{d}}^{0}\left(P_{0}, x\right) \cdot a_{\mathrm{d}}(x, t) \\
\left(a_{\mathrm{d}}\right)_{t=t_{\mathrm{m}}}=1-\int_{0}^{t=t_{\mathrm{m}}} \phi_{\mathrm{d}}\left(P_{0}, x\right) \mathrm{d} t
\end{gathered}
$$

The " $k$ " and "d" subscripts distinguish the kinetic and diffusion-limited regions.

The ratio of the reaction rates under kinetic and diffusion control will depend on the initial rates and on the values of the activity functions:

$$
\left(\frac{r_{\mathrm{k}}}{r_{\mathrm{d}}}\right)_{t}=\left(\frac{r_{\mathrm{k}}^{0}}{r_{\mathrm{d}}^{0}}\right) \cdot\left(\frac{\left(a_{\mathrm{k}}\right)_{t}}{\left(a_{\mathrm{d}}\right)_{t}}\right)
$$

Initially, when the catalyst is not deactivated, $\left(a_{\mathrm{k}}\right)_{0}=1$; $\left(a_{\mathrm{d}}\right)_{0}=1$, and

$$
\left(\frac{r_{\mathrm{k}}}{r_{\mathrm{d}}}\right)_{t \rightarrow 0}=\left(\frac{r_{\mathrm{k}}^{0}}{r_{\mathrm{d}}^{0}}\right)>1
$$

With deactivation, the values of $\left(a_{\mathrm{k}}\right)$ and $\left(a_{\mathrm{d}}\right)$ decrease at different rates, depending on the $\phi_{\mathrm{k}}(u)$ and $\phi_{\mathrm{d}}(u)$ functions. Accordingly, the $\left(r_{\mathrm{k}}(t) / r_{\mathrm{d}}(t)\right)$ ratio will change.

In the case that

$$
\phi_{\mathrm{d}}(t)<\phi_{\mathrm{k}}(t)
$$

the decrease of catalyst activity will be slower under diffusion control, and consequently 


$$
\left(a_{\mathrm{d}}\right)_{t_{\mathrm{a}}}>\left(a_{\mathrm{k}}\right)_{t_{\mathrm{a}}}
$$

It follows from Eq. (38) that the ratio of activities under kinetic and pore diffusion control will decrease with time on stream. In such a case, at $t_{\mathrm{c}}$, the inequality

$$
\left(\frac{\left(a_{\mathrm{k}}\right)_{t_{\mathrm{c}}}}{\left(a_{\mathrm{d}}\right)_{t_{\mathrm{c}}}}\right)<\left(\frac{\left(a_{\mathrm{k}}\right)_{0}}{\left(a_{\mathrm{d}}\right)_{0}}\right)
$$

may lead to the relationship

$$
\left(\frac{\left(a_{\mathrm{d}}\right)_{t_{\mathrm{c}}}}{\left(a_{\mathrm{k}}\right)_{t_{\mathrm{c}}}}\right) \geq\left(\frac{r_{\mathrm{k}}^{0}}{r_{\mathrm{d}}^{0}}\right)
$$

Under these conditions, the rate of the reaction under diffusion control may equilize, or even exceed the reaction rate attained after the same period of time under similar operation conditions in the absence of diffusion resistance

$$
\left(r_{\mathrm{d}}\right)_{t \geq t_{\mathrm{c}}} \geq\left(r_{\mathrm{k}}\right)_{t \geq t_{\mathrm{c}}}
$$

Equations (39)-(41) indicate the possibility that the reaction system may attain a point at which the effect of diffusion retardation of the deactivation process will be much stronger than diffusion retardation of the reaction. Therefore the product yield will be higher and catalyst life will be prolonged under diffusion control. Obviously, such a possibility may be the case under certain conditions depending on the reaction mechanism, on the mechanism of the coke formation reaction, and on the operation conditions. In a series of works [34-36], we have developed the concept that modeling the deactivation kinetics of processes affected by intraparticle diffusion should include the changes of the chemical reaction rates and the changes of the catalyst deactivation rate caused by diffusion resistance.

To show the influence of diffusion retardation on both catalytic reaction and catalyst deactivation, we will derive the kinetic description in terms of conversion. By applying equations proposed by Pshezhetsky and Rubinstein [37], we can account for the influence of diffusion on the reaction rate of the main reaction and of the catalyst deactivating reactions. We obtain as a first approximation the equations

$$
\begin{aligned}
& r_{\mathrm{d}}(x)=\frac{\sqrt{2 D_{1} P_{0}}}{R_{\mathrm{g}}} \sqrt{\int_{x_{\mathrm{s}}}^{x_{\mathrm{eq}}} r_{\mathrm{k}}(x) \mathrm{d} x} \\
& \varphi_{\mathrm{d}}(x)=\frac{\sqrt{2 D_{2} P_{0}}}{R_{\mathrm{g}}} \sqrt{\int_{x_{\mathrm{s}}}^{x_{\mathrm{eq}}} \phi_{\mathrm{k}}(x) \mathrm{d} x}
\end{aligned}
$$

$R_{\mathrm{g}}$ denotes the effective radius of the catalyst particle, $D_{1}$ and $D_{2}$ stand for the effective diffusion coefficients of the key species in the main and deactivating reactions. Eq. (42) gives the rate of the reaction under diffusion control, while Eq. (43) gives the rate of the coking reaction under diffusion control; $r_{\mathrm{k}}$ and $\phi_{\mathrm{k}}$ are the rate expressions for these processes under kinetic control.

The analysis of the deactivation-conversion curves can indicate the influence of deactivation on the reaction rate at high or low conversions. Figure 2 shows curves for the dependence of normalized coke formation rates on conversion calculated for model reactions proceeding under kinetic and diffusion control via parallel-parallel and parallel-consecutive deactivation schemes. The simulated curves correspond to reasonable values of the rate coefficients for the assumption that the rate of the coke formation reaction is an order of magnitude lower than the rate of the process. For the parallel-parallel mechanism of coking, the coke formation rate is low at high conversion, and its trend is to increase with decreasing conversion. It can be concluded that in this mechanism of coke formation, operating the process at high conversions may suppress the generation of coke agents to a certain degree.

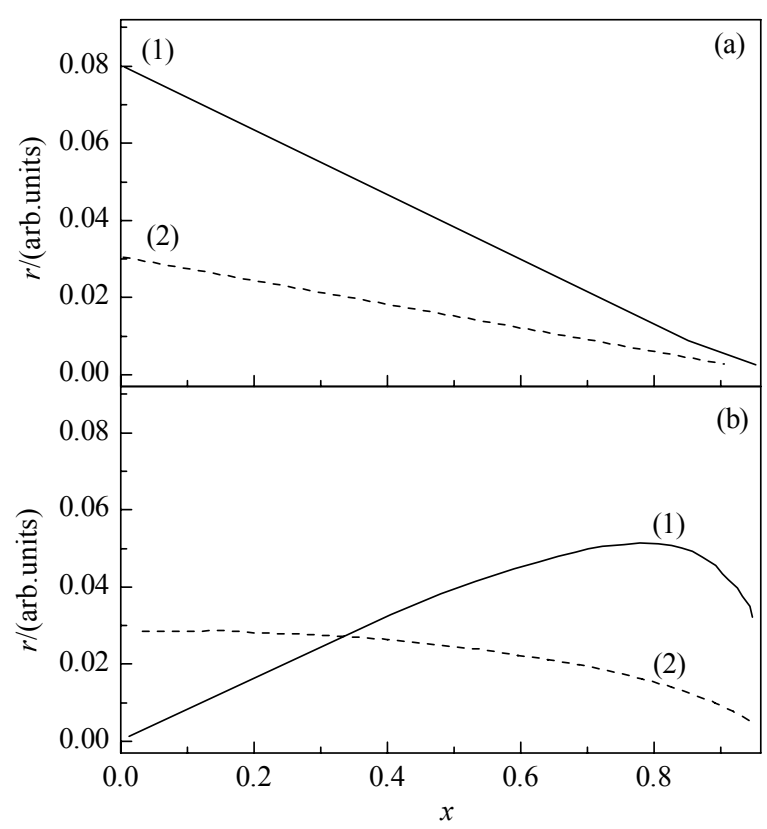

Fig. 2. Conversion curves of the coking rates calculated for model reactions proceeding via parallel-parallel (a) and parallel-consecutive (b) mechanisms under kinetic (1) and diffusion (2) control [26].

In the case of the parallel-consecutive mechanism of coke formation, the curves of the deactivation rate pass through a maximum in the kinetic region, and smoothly decrease with conversion under diffusion control. For this type of mechanisms, the trends are that in the domain of low conversion, the formation of coke precursors will be slower in the absence of diffusion restrictions. In contrast, at higher conversions, diffusion resistance may lead to lower of the rate of coke generation.

The characteristics of the deactivation-conversion dependencies determine the evolution of the reaction processes under kinetic or diffusion control. The plots in Fig. 3 show the simulated evolution of the total reaction rates in the absence and presence of diffusion restrictions. In the 


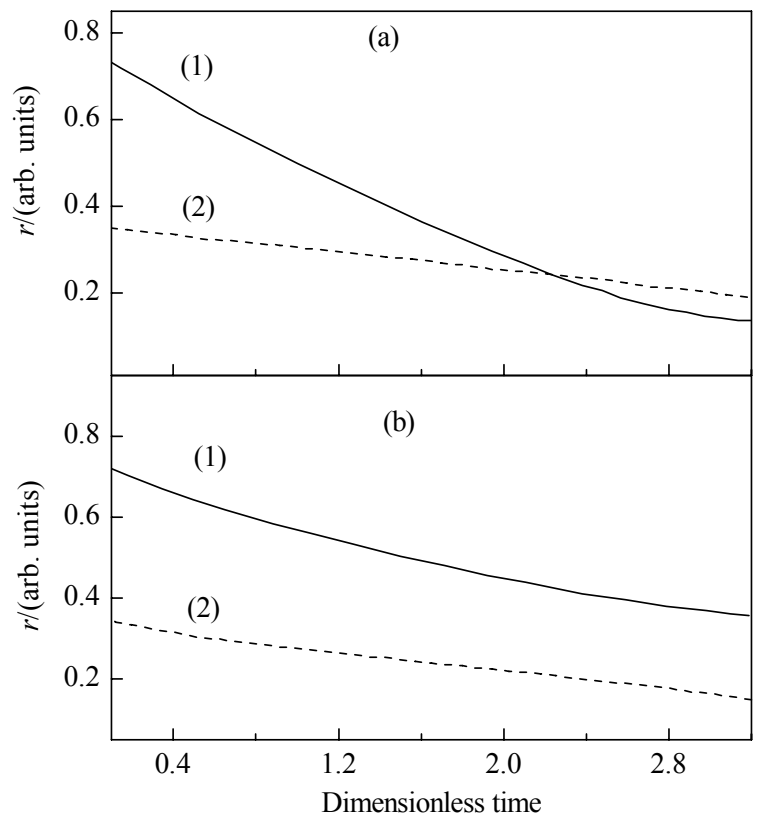

Fig. 3. Conversion curves of the reaction rate under conditions of kinetic (1) and diffusion (2) control calculated for model reactions proceeding via parallel-consecutive (a) and parallel-parallel (b) mechanisms [26].

simulations, the size of the catalyst particles was the only factor determining diffusion limitation.

For the illustration of the ideas described above, we performed the analysis of two important industrial processes, namely: (i) hydrogenation of nitrobenzene to aniline over a commercial $\mathrm{Cu}$ catalyst; and (ii) dehydrogenation of isoamylenes into isoprene over a commercial calcium-nickel-phosphate catalyst.

\subsection{Hydrogenation of nitrobenzene to aniline over a commercial $\mathrm{Cu}$ catalyst}

The kinetic description of the experimental data obtained [38] on small catalyst particles follows separable deactivation kinetics. The kinetic model of nitrobenzene hydrogenation to aniline and the change of the catalyst activity in the kinetic regime are expressed, respectively, by Eqs. (44-a) and (44-b):

$$
\begin{gathered}
r_{\mathrm{k}}(t)=\frac{k_{1} P_{\mathrm{nb}} P_{\mathrm{H}}}{1+k_{2} P_{\mathrm{nb}}} \cdot a_{\mathrm{k}}(t) \\
a_{\mathrm{k}}(t)=1-K\left\{\left(\frac{P_{\mathrm{nb}}}{P_{\mathrm{H}}}\right)_{t}-\left(\frac{P_{\mathrm{nb}}}{P_{\mathrm{H}}}\right)_{0}\right\}
\end{gathered}
$$

Here, $r_{\mathrm{k}}$ is the kinetic rate of the hydrogenation reaction in the absence of deactivation and diffusion limitations, $a_{\mathrm{k}}(t)$ is the activity function, $P_{\mathrm{nb}}$ and $P_{\mathrm{H}}$ are the partial pressures of nitrobenzene and hydrogen, $k_{1}$ and $k_{2}$ and $K$ are rate coefficients for the hydrogenation and deactivation reactions.
The activity function takes into account the experimental observations that the initial reactant nitrobenzene is responsible for coke formation. Therefore, a high nitrobenzene partial pressure promotes coke formation, while a high partial pressure of $\mathrm{H}_{2}$ depresses coke formation. For this reason, the process is carried out with a large excess of hydrogen: the mole ratio of nitrobenzene to hydrogen in the initial reaction mixture is 1:15. According to the proposed reaction scheme, $\mathrm{H}_{2}$ from the gas phase combines successively with the intermediates generated from adsorbed nitrobenzene. Parallel to this, the same intermediates give rise to coke precursors.

On applying the modified formula of Pshezhetsky and Rubinstein [37], the following relationships were obtained for the initial diffusion-controlled rate of nitrobenzene hydrogenation over fresh catalyst particles.

$$
r_{\mathrm{dif}}=\frac{k_{1}}{R_{\mathrm{g}}}\left(\frac{2 D_{\mathrm{eff}} P_{\mathrm{H}}}{k_{2}}\left(P_{\mathrm{nb}}-\ln \left(1+k_{2} P_{\mathrm{nb}}\right)\right)\right)^{0.5}
$$

The experimental data obtained on catalyst particles of industrial size confirmed these relationships with an accuracy of $11.8 \%$

On applying the modified formula of Pshezhetsky and Rubinstein, the following relationships were obtained for the hydrogenation rate $r_{\mathrm{d}}^{*}(t)$ and the change of the activity function $a_{\mathrm{d}}^{*}(t)$ taking place under diffusion control:

$$
\begin{gathered}
r_{\mathrm{d}}^{*}(t)=\frac{k_{1}}{R_{\mathrm{g}}}\left(\frac{2\left(D_{\text {eff }}\right)_{t} P_{\mathrm{H}}}{k_{2}}\left(\left(P_{\mathrm{nb}}\right)_{t}\left(\ln \left(1+k_{2}\left(P_{\mathrm{nb}}\right)_{t}\right)\right)\right)^{0.5} \cdot a_{\mathrm{d}}^{*}(t)\right. \\
a_{\mathrm{d}}^{*}(t)=1-\kappa_{\mathrm{d}}^{*}\left\{1-\frac{\left(P_{\mathrm{nb}}\right)_{t}}{\left(P_{\mathrm{nb}}\right)_{t=0}}\right\}
\end{gathered}
$$

The experimental data obtained on catalyst particles of industrial size confirmed these relationships with an accuracy of $6.9 \%$.

In the kinetic region the deactivation is a function of the partial pressures of both nitrobenzene and hydrogen, whereas under diffusion control, it is a function only of the nitrobenzene concentration. Due to their small size, hydrogen molecules can penetrate into the catalyst pores more easily than nitrobenzene molecules, and for this reason, the concentration gradient of hydrogen along the particle radius can be considered negligible compared with that of nitrobenzene. Correspondingly, the probability for the formation of coke precursors along the catalyst particle radius will depend mainly on the rate of nitrobenzene diffusion, which on turn is proportional to its gas phase concentration.

This example shows how diffusion resistance can modify the activity function during deactivation of the catalysts. As for use of the catalyst, both simulation results and experimental observations indicate that catalyst deterioration will be more pronounced under diffusion control. 


\subsection{Dehydrogenation of isoamylenes into isoprene}

The situation is different when product molecules participate in the generation of coke precursors. Under some conditions, in the course of deactivation, the process can get to a point where the rate in the presence of diffusion restrictions exceeds the rate in their absence. This conclusion has been experimentally confirmed for the isoprene production processes described below.

From experimental data [39] obtained on small catalyst particles for the dehydrogenation of isoamylenes over a commercial nickel-calcium-phosphate catalyst, the dehydrogenation rate $r_{\mathrm{k}}$ and the rate of coke formation $r_{\mathrm{k}}^{*}$ are described by

$$
\begin{gathered}
r_{\mathrm{k}}=\frac{k P_{0}(1-x)}{1+Q+\alpha P_{0} x} \\
r_{\mathrm{k}}^{*}=P_{0}\left\{\frac{k_{2}+k_{3} x}{1+Q+\alpha P_{0} x}+\frac{k_{4}+k_{5} x}{\sqrt{1+Q+\alpha P_{0} x}} \cdot Q \exp (-\mu Q)\right\}
\end{gathered}
$$

for $Q=C^{2 / 3} ; C$-coke deposited per gram-catalyst.

The coke formed in the course of this process comes from the product (isoprene) and its adsorbed forms (series-consecutive mechanism).

The concentration of coke precursors is related to the product concentration, which is lower under diffusion control. The analysis performed concluded that the deactivation process will be less pronounced under conditions of diffusion control. The intraparticle diffusion effects actually cause a decrease in the rate of the chemical reaction but at the same time, retard the deactivating reaction. The superposition of these opposite retardation effects may result in their mutual compensation. Moreover, the effective rate of the process with intraparticle resistance may reach a point where it even exceeds the rate of the chemical reaction on the decaying catalyst in the kinetic region.

To verify this suggestion, we applied the modified formula of Pshezhetsky and Rubinstein to obtain the specific dependencies of the main reaction (Eq. (59)) and coke formation rates (Eq. (51)) under diffusion control as functions of conversion:

$$
\begin{aligned}
& r_{\mathrm{d}}=\frac{\sqrt{2 D_{1} P_{0}}}{R_{\mathrm{g}}} \sqrt{\int_{x_{\mathrm{s}}}^{x_{\mathrm{eq}}} r_{\mathrm{k}}(x) \mathrm{d} x} \\
& r_{\mathrm{d}}^{*}=\frac{\sqrt{2 D_{2} P_{0}}}{R_{\mathrm{g}}} \sqrt{\int_{x_{\mathrm{s}}}^{x_{\mathrm{eq}}} r_{\mathrm{k}}^{*}(x) \mathrm{d} x}
\end{aligned}
$$

The expressions for $r_{\mathrm{k}}(x)$ and $r_{\mathrm{k}}^{*}(x)$ are taken from Eqs. (48) and (49) correspondingly. By using Eqs. (50) and (51), we calculated the change of conversion for the following two cases:

(i) The catalytic reaction takes place in the kinetic re- gion over catalyst particles of size $0.05 \mathrm{~cm}$. The results are plotted in Fig. 4(a) as the full line.

(ii) The catalytic reaction takes place in the diffusioncontrolled region over catalyst particles of size $1.5 \mathrm{~cm}$. The effective diffusion coefficients in Eqs. (50) and (51) have the following values $D_{1}=0.004 \mathrm{~cm}^{2} / \mathrm{s}, D_{2}=0.0001 \mathrm{~cm}^{2} / \mathrm{s}$. The results are plotted in Fig. 4(a) as the dotted line.

It can be seen from the calculated conversion-time on stream curves shown that the decrease of conversion is steeper in the kinetic region, and after some time, the conversion obtained under diffusion control exceeds that in the kinetic region.

The possibility of this behavior of a catalytic system was found from the theoretical analysis of kinetic models (50) and (51) and experimental observations. Laboratory tests confirmed the existence of the predicted effect [36]. The experimental curves obtained in a laboratory flow reactor are presented in Fig. 4(b). The data obtained in the kinetic region is plotted as the full line. The data obtained under the diffusion limited regime is presented by the dotted line.

The theoretical analysis and experimental results showed that in some cases it is better to carry out catalytic processes accompanied by catalyst deactivation under the diffusion limited regime in order to get a higher yield and longer catalyst life.
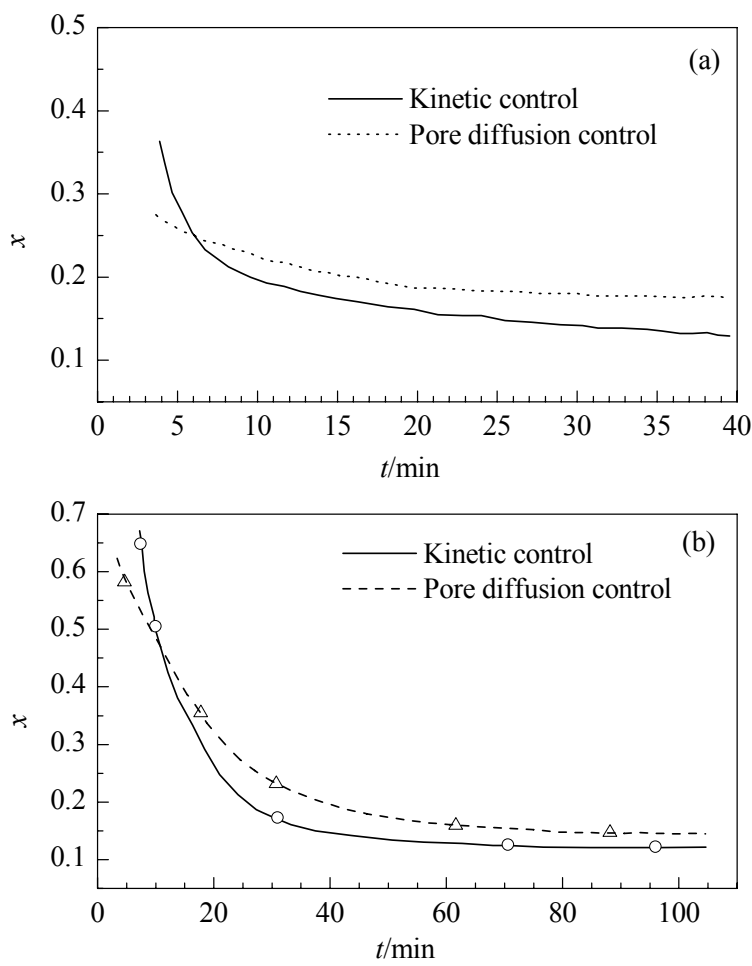

Fig. 4. Evolution of the conversion $(x)$ in the course of amylene dehydrogenation in a flow reactor at $680{ }^{\circ} \mathrm{C}$ under conditions of kinetic and pore diffusion control. (a) Calculated curves; (b) Experimental curves [36]. 


\subsection{Optimization of processes accompanied by catalyst deactivation}

Catalyst deactivation forces a considerable number of industrial processes affected by deterioration of the catalyst to be operated in cycles, with each cycle consisting of a work period, $\tau$, and a regeneration period, $\tau_{0}$.

It is obvious that the duration of the work period $\tau$ for most petrochemical processes is determined by the permissible range of activity decrease caused by coke formation. The typical example is the process of dehydrogenation of amylenes to isoprene. In this manufacture, the work period of $20 \mathrm{~min}$ is followed by a regeneration period of $10-15 \mathrm{~min}$ duration. The plots in Fig. 5 show the increase of isoprene yield with time on stream for equal work and regenerating periods for a catalyst under kinetic and diffusion limited control, i.e. $\tau_{\mathrm{k}}=\tau_{\mathrm{d}}=20 \mathrm{~min} ; \tau_{0}=10 \mathrm{~min}$. The amount of coke deposited by the end of the work cycle was about 25 $\mathrm{mg}$ per gram catalyst in the kinetic region, and $20 \mathrm{mg}$ per gram catalyst in the diffusion limited region. These results show the possibility of prolonging the working time of the catalyst by carrying out the process under diffusion control, where the work period is the time used for dehydrogenation until a critical decrease of the conversion is attained. Thus, if the critical value of conversion is 0.2 , the work period in the kinetic region would be $\tau_{\mathrm{k}}=20 \mathrm{~min}$, while the work period under diffusion control would be $\tau_{\mathrm{d}}=60 \mathrm{~min}$. Therefore, for processes over fast deactivating catalysts, it is possible to improve the catalyst performance by performing the process under the diffusion limited regime. Practically, this can be realized by the use of larger catalysts particles.

The plots in Fig. 6 show the predicted increase with time of the isoprene yield in the kinetic and in the pore diffusion limited regions with the suggested assumptions.

Evidently, for processes in which the product molecules from the gas phase are responsible for coke formation, it is

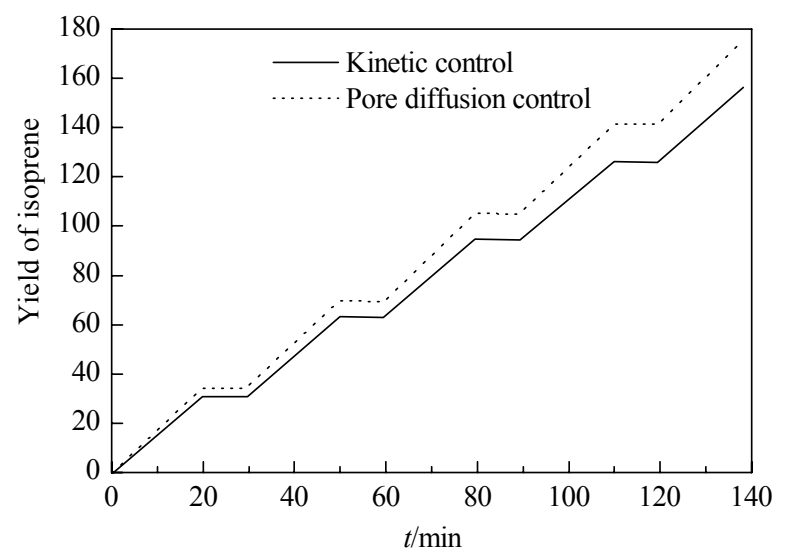

Fig. 5. Isoprene yield accumulated in the course of time under conditions of kinetic and pore diffusion control calculated for $\tau_{\mathrm{k}}=\tau_{\mathrm{d}}=20$ $\min ; \tau_{0}=10 \min [35]$.

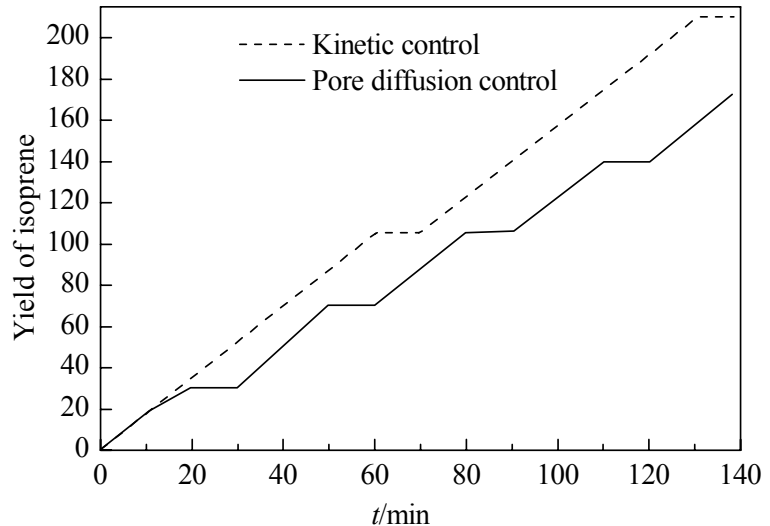

Fig. 6. Isoprene yield accumulated against time under conditions of kinetic and pore diffusion control calculated for $\tau_{\mathrm{k}}=20 \mathrm{~min}, \tau_{\mathrm{d}}=60$ $\min , \tau_{0}=10 \min [35]$.

advantageous to run the process with larger catalyst particles, in order to reduce the deterioration of catalyst activity. In cases when intraparticle diffusion resistance proves to be advantageous, this should be taken into account in the process design.

\subsection{Coking profiles inside the catalyst pores}

The analysis of the combined effects of catalyst deactivation and mass transfer should pay attention to the distribution of coke deposits along the radius of the catalyst particle. Due to the key contribution of surface intermediates to coke formation, when modeling the deactivation kinetics of catalytic processes over large catalyst particles, not only should the diffusion processes be taken into account, but also the intrinsic distribution of surface intermediates along the radius of the catalyst pellet. Considering a single-ended pore, the kinetic description of the process is a set of coupled nonlinear differential equations relating mass transfer and deactivation kinetics with the position inside the catalyst particle [40].

$$
\begin{gathered}
\frac{\mathrm{d} P}{\mathrm{~d} t}=\frac{D}{\varepsilon}\left\{\frac{\partial}{\partial \rho}\left(\frac{\partial P}{\partial \rho}\right)+\frac{2}{\rho}\left(\frac{\partial P}{\partial \rho}\right)-\frac{r_{j}(P,(\sigma)}{\varepsilon}\right\} \\
\frac{\mathrm{d} \sigma}{\mathrm{d} t}=\phi_{j}(P, \sigma(t))
\end{gathered}
$$

Each of the mechanisms listed in Table (1) can result in certain specific features in the profile of coke distribution inside the particle pores. In the case of when the coke precursors arise through surface transformations of intermediates of type [AZ], or by contribution of the feed species, the blocked fraction of the pores is mostly located in the areas adjacent to the pellet surface. Deposition of coke at the entrance of the pores has a very harmful influence on catalyst activity, as the most efficient areas of the active surface are the first to be blocked. For these cases, it is preferable to use 
small catalyst particles to carry out the process under kinetic regime, thus avoiding the harm caused by the pore blockage.

In case when the coke precursors originate through surface transformations of intermediates of the type [MZ], the maximum of coke deposits is expected to be situated near the core of the particle.

In case interactions of surface intermediates with desorbed product molecules play a key role in the generation of coke precursors, the deactivation function is humpshaped along the particle radius. At the beginning of the process, the inner surface area adjacent to the pore mouth is less affected by coke formation. Thus, the most efficient catalyst surface is expected to keep its activity to a higher degree under diffusion control. For optimal process design, it can be concluded that using catalyst pellets of a size that provides diffusion limitation will hinder the deteriorative action of coking.

\section{Problems of selectivity}

The influence of diffusion retardation on process selectivity, as well as on selectivity changes caused by deactivation has been separately examined in numerous studies. Yet, little attention has been paid to the joint effects of mass transfer and coke formation on the selectivity of complex reactions. Here, we briefly discuss their combined action in connection with the mechanism.

From the standpoint of kinetics, the problem is the functional dependence of selectivity on conversion. The analysis performed [41] indicated that, depending on the reaction mechanism, the selectivity may either decrease or increase with the conversion. To get more details on this behavior, we examine the calculated curves of selectivity versus conversion, or so called "selectivity-conversion curves". These curves are different for different reaction schemes.

We denote by $A$ the initial reactants, by $M$ the target product, by $\mathrm{D}$ the side product, and by $(\mathrm{PZ})$ the coke precursors. In general, it is necessary to include that the side products and/or coke precursors exist as a result of interactions between surface intermediates (denoted as $[\mathrm{AZ}]_{j}$ or $[\mathrm{MZ}]_{j}$ ) and reactants from the gas phase. We consider three mechanism types with side products.

Fork selectivity type: the primary intermediates undergo a succession of surface transformations in the course of which two branching routes arise:

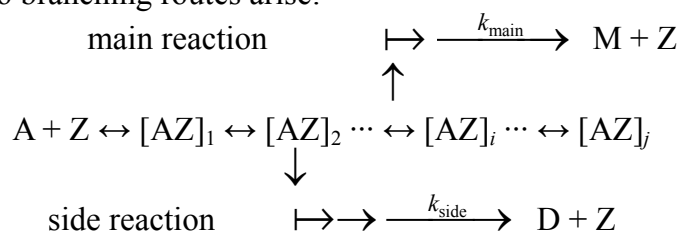

where $k_{\text {main }}$ and $k_{\text {side }}$ denote the rate coefficients of the main and side reaction steps, respectively. Generally, processes that follow this mechanism are characterized as isokinetic: the selectivity does not depend on the conversion [42], both in the absence of deactivation, and when coke precursors come from side products.

Competitive-parallel selectivity type: the formation of desired and side products is through competing interactions of the initial species with free catalyst sites or some of the intermediates, respectively:

$$
\begin{array}{r}
\mathrm{A}+\mathrm{Z} \leftrightarrow[\mathrm{AZ}]_{1} \leftrightarrow[\mathrm{AZ}]_{2} \cdots \leftrightarrow[\mathrm{AZ}]_{j} \stackrel{k_{\text {main }}}{\longrightarrow}[\mathrm{MZ}]_{j+1} \\
\leftrightarrow \cdots \leftrightarrow[\mathrm{MZ}]_{\mathrm{m}} \leftrightarrow \mathrm{M}+\mathrm{Z} \text { main reaction } \\
\mathrm{A}+[\mathrm{AZ}]_{\mathrm{s}} \stackrel{k_{\text {side }}}{\longrightarrow} \mathrm{D}+\mathrm{Z} \text { side reaction (55) }
\end{array}
$$

Consecutive selectivity type: the side product is produced through interactions of surface intermediates with desorbed molecules of the target product:

$$
\begin{aligned}
& \mathrm{A}+\mathrm{Z} \leftrightarrow[\mathrm{AZ}]_{1} \leftrightarrow[\mathrm{AZ}]_{2} \cdots \leftrightarrow[\mathrm{AZ}]_{j} \stackrel{k_{\text {main }}}{\longrightarrow}[\mathrm{MZ}]_{j+1} \\
& \leftrightarrow \cdots \leftrightarrow[\mathrm{MZ}]_{\mathrm{m}} \leftrightarrow \mathrm{M}+\mathrm{Z} \text { main reaction } \\
& \mathrm{M}+[\mathrm{AZ}]_{j} \stackrel{k_{\text {side }}}{\longrightarrow} \mathrm{D}+\mathrm{Z} \text { side reaction }
\end{aligned}
$$

In the coke formation mechanism, for clarity, the various complicated mechanistic patterns can be reduced to simple terms by considering just the routes with major contribution to coking:

$$
\begin{aligned}
\mathrm{A}+[\mathrm{AZ}]_{j} & \rightarrow(\mathrm{PZ}) \rightarrow \text { coke } \\
\mathrm{A}+[\mathrm{MZ}]_{j} & \rightarrow(\mathrm{PZ}) \rightarrow \text { coke } \\
\mathrm{M}+[\mathrm{AZ}]_{j} & \rightarrow(\mathrm{PZ}) \rightarrow \text { coke } \\
\mathrm{M}+[\mathrm{MZ}]_{j} & \rightarrow(\mathrm{PZ}) \rightarrow \text { coke } \\
\mathrm{D}+[\mathrm{AZ}]_{j} & \rightarrow(\mathrm{PZ}) \rightarrow \text { coke } \\
\mathrm{D}+[\mathrm{MZ}]_{j} & \rightarrow(\mathrm{PZ}) \rightarrow \text { coke } \\
\mathrm{M}+\mathrm{Z} & \rightarrow(\mathrm{PZ}) \rightarrow \text { coke } \\
\mathrm{D}+\mathrm{Z} & \rightarrow(\mathrm{PZ}) \rightarrow \text { coke }
\end{aligned}
$$

By coupling Schemes (54), (55), or (56) with each of these simplified coke formation mechanisms, we get a variety of probable types of complex reactions. In each particular case, the interrelation between the selectivity function and conversion is specific, and depends on the mechanistic features. The analysis of the dependence of the selectivity functions on conversion provides interesting information about the behavior of the system during deactivation (i.e., decrease of conversion). Calculations performed in [41] consider the changes of selectivity of processes accompanied catalyst deactivation. It was shown that the selectivity may either decrease or increase, depending on the reaction mechanism. For more insight into this matter, we simulated the performance of each of the model reactions under kinetic and internal diffusion limited regime [41]. The only factor determining the region of control was the size of the catalyst particles. According to the analysis, the changes are more pronounced under kinetic control. These calculations may have practical use for elucidating how to reduce the harmful effects of deactivation on the yield of the desired products. In case the process mechanism is conductive to decrease of selectivity with the drop of conversion kinetic 
region, while under diffusion control, the selectivity is higher and remains less affected, it may be advantageous to perform the process on catalyst particles that ensure intraparticle resistance regime. In case the reaction mechanism leads to an increase of the selectivity in the course of deactivation, it is recommended to run the process with small catalyst particles.

\section{Reactions proceeding through the participation of several site types}

There is a special class of catalysts in which more than one type of active sites participate in the reaction. The detailed study of these catalysts is important because bifunctional catalysts are used in many industrial processes. Distinct types of sites may differ by configuration, nature, adsorption ability, contribution to different reaction routes, vulnerability, etc [43-45]. Each of these types are affected differently by various deactivation factors. The initial ratio of the different types of sites depends on the catalyst, preparation procedure, and reaction conditions. Thus, it is believed that coordinately unsaturated sites (called CUS sites) and Brönsted acid sites of HDS and HDN catalysts can inter-convert into each other, depending on the $\mathrm{H}_{2} \mathrm{~S}$ concentration in the reaction mixture [12,43-45]. Raising the $\mathrm{H}_{2} \mathrm{~S}$ concentration over a $\mathrm{NiMo} / \mathrm{Al}_{2} \mathrm{O}_{3}$ catalyst transforms anionic vacancies, on which hydrogenation occurs, into Brönsted acid sites responsible for hydrogenolysis, which are less vulnerable to poisoning from N-compounds. Starting from different initial surface concentrations, each type of sites can evolve during the catalytic process in its own way, depending on its vulnerability under the action of the various deactivating factors.

The complexity of such catalytic systems is associated not only with the variety of interactions and species involved in the reaction network, but also with the deactivation functions of each site type in the course of the process. Usually two or three types of sites participate in these processes. The particular functions describing the changes in the contribution of the $j$-th site type can be specified as

$$
\phi_{j}(u)=\partial a_{j} / \partial u
$$

Numerous examples in the literature reported that bimetallic catalysts manifest a higher resistance to coke [46]. This may be associated with change of the electron density and/or geometric effects. Coke precursors originate predominantly from intermediates strongly bound to the catalyst surface [25]. Promoting additives such as Sn, In, and others may modify the adsorption properties of the catalyst [46-49].

Evidence in the literature show that coking is a structure sensitive process requiring large ensembles or catalytic clusters [50]. The introduction of a second metal may result in an increased dispersion, and hence a decrease in the area of the active phase islands, thus reducing the number of large ensembles that facilitate coke formation. At the same time, the main reaction can be either structure insensitive (e.g. dehydrogenation requiring $1 \mathrm{Pt}$ atom), or require ensembles of different configuration or size, and therefore, exert different structure sensitivity. By taking this into account and using of the model suggested by Beeckman and Froment $[51,52]$, we developed an approach to describe the deactivation behavior of catalytic systems with several types of active sites [53]. The original model [51,52] considers the fraction of active sites $\varphi(t)$ as the product of the probability $H(t)$ that a site is accessible, and the conditional probability $S(t)$ that the site is not out of action at time $t$ :

$$
\varphi(t)=S(t) \cdot H(t)
$$

For catalytic reactions with one type of sites, the $\varphi(t)$ function coincides with the activity function $a(t)$ defined by (2), the $S(t)$ function with the fraction $\sigma(t)$ of unblocked surface active sites. In the case of reaction systems involving several types of sites, the non-uniformity of the catalyst surface can lead to various critical effects [54]. Special models must be derived for processes involving active sites of different structure, action, and accessibility. By extending the model of Beeckman and Froment, the activity function can be defined as a sum of the "individual activity" functions ascribed to particular types of active sites.

The overall catalyst activity $\varphi(u)$ of multifunctional catalysts can be defined as:

$$
\varphi(u)=\sum \varphi_{j}(u)=\sum H_{j} \sigma_{j}(u)
$$

where the $\varphi_{j}(u)$ terms denote the contribution of each type of sites, $\sigma_{j}(u)$ the working fraction of the $j$-th site type, $H_{j}$ the probability that the site is accessible, $j$ is equal to 1,2 , or 3 . The $\sigma_{j}(u)$ functions may evolve in different ways for types of sites requiring different surface ensembles. Accordingly, some of the reaction routes may be more strongly affected than others.

Defining the overall deactivation function $\Phi(u)$ as the change of the overall activity $\varphi(u)$, for catalysts with a bi-dispersed pore structure we get

$$
\begin{gathered}
\Phi(u)=\frac{\partial \phi(u)}{\partial u}=H_{1} \frac{\partial \sigma_{1}}{\partial u}+H_{2}\left\{\frac{\partial \sigma_{2}}{\partial u}+\frac{\partial \sigma_{3}}{\partial u}\right\}+ \\
\sigma_{1}(u) \frac{\partial H_{1}}{\partial u}+\left\{\sigma_{2}(u)+\sigma_{3}(u)\right\} \frac{\partial H_{2}}{\partial u}
\end{gathered}
$$

From this relationship, various trends in the evolution of the catalytic system can appear depending on the quantitative changes of $\sigma_{1}, \sigma_{2}, \sigma_{3}$, and $H_{j}$ caused by changes of $u$ ( $u$ may be time, conversion, coke, poison concentration, or any other deactivation factor). The $\sigma_{j}$ terms can change in different way for site types requiring different surface ensembles.

The different site types can be vulnerable in different 
ways under the influence of the reaction medium. Thus, if $u$ stands for conversion, in the general case $\partial \sigma_{i} / \partial u \neq \partial \sigma_{j} / \partial u$, depending on the origin of the deactivating agents. The evolution of the catalytic system will be ruled by the $\left(\partial \sigma_{j} / \partial u\right)$ trend which satisfies the condition:

$$
\partial \sigma_{j} / \partial u>\partial \sigma_{m} / \partial u \geqslant \partial \sigma_{n} / \partial u
$$

A step change in the deactivation law may be observed in the course of the process, if the system gets to a point where

$$
\partial \sigma_{m} / \partial u \geqslant \partial \sigma_{j} / \partial u
$$

As an example we have the process of butane dehydrogenation over Pt/alumina catalysts promoted by In or $\mathrm{Sn}$, which were studied in detail in [55-57]. According to the observations reported in $[55,56]$, initially, the dehydrogenation reaction proceeded in a steady state regime; the accompanying coke formation did not affect catalyst activity. When the coke deposits attained a critical amount $\left(C^{*}\right)$, the coke formation rate abruptly dropped, while the reaction kept its quasi-stationary regime. This lasted for $7 \mathrm{~h}$ on the unpromoted Pt catalyst, $15 \mathrm{~h}$ on the In-promoted, and $20 \mathrm{~h}$ on the Sn-promoted samples. During this phase of the process, coke formation was proceeding at a low constant rate, until the coke amount attained a second critical value $C^{* *}$ (respectively, $1.8 \mathrm{wt} \%, 2.3 \mathrm{wt} \%$, and $6.8 \mathrm{wt} \%$ for the different samples). After that threshold, the rate of dehydrogenation began to slow down, with a linear dependence on the amount of coke exceeding $C^{* *}$. Detailed studies of these catalysts performed elsewhere [58] by infrared (IR) spectroscopy, differential thermal analysis (DTA), electron paramagnetic resonance (EPR), X-ray photoelectron spectroscopy (XPS), and other methods have shown that the presence of promoters leads to smaller sizes of active-phase islands. The data supplied evidence that the additives reduce the adsorption energy of strongly adsorbed molecules and facilitate the migration of coke precursors formed inside the metal-phase islands towards the interface with the support. The observed features of the process $[55,56]$ can be adequately described within the framework of a model that assume the participation of three types of active sites: single Pt centers of strong adsorption affinity that facilitate dehydrogenation, catalytic clusters (ensembles involving a number of Pt (and possibly Sn or In) surface atoms in a proper configuration) that facilitate cracking, and centers of weaker adsorption ability at the interfaces $\mathrm{Pt} /$ carrier or $\mathrm{Pt} /$ promoter that facilitate dehydrogenation.

The intermediates adsorbed on the interfaces are weakly bound. This gives the chance for the dehydrogenation products formed on them to desorb rather than to become involved in condensation processes.

Two types of precursors responsible for coke formation were postulated: (i) precursors arising from intermediates strongly adsorbed on the catalyst metal clusters; (ii) precur- sors located at the metal-carrier boundary sites. The (ii)-type precursors may be formed from the migration of the first type of precursors from the internal area of the metal clusters or by direct adsorption of hydrocarbon on the interfacial active centers. The coke precursors located on the different types of sites influence in different ways the evolution of the reaction system [57]. The relationships obtained from the suggested model qualitatively explained the experimentally observed stepwise character of the deactivation kinetics, as well as other promoter-induced effects. Additional details on this matter can be found in the original works [55-57].

The essential aspect of the geometric effect is that the various types of active sites often differ by the number of adjacent surface atoms in a proper configuration, which makes them vulnerable to coking in different ways. The contributions of clusters with different composition and geometry lead to different ways and rates of coke formation. Consider a catalytic system in which a structure insensitive (e.g. dehydrogenation) step on single surface atoms is accompanied by structure sensitive (cracking, isomerization, etc) reactions requiring catalytic clusters consisting of nano-entities of $M$ surface atoms in a proper configuration. Let us denote by $\varphi_{1}$ and $\varphi_{2}$ the contributions, respectively, of single sites and of catalytic clusters to the overall activity, by $\sigma_{1}$ the surface concentration of single active atoms, and by $\sigma_{2}$ the surface concentration of the catalyst clusters. We can write:

$$
\begin{gathered}
\varphi_{1}(u)=k_{1} \sigma_{1}(u) \\
\varphi_{2}(u)=k_{2} \sigma_{2}(u)
\end{gathered}
$$

In the simplest case, the surface concentration of catalytic clusters is related to the density of single active sites through the relation

$$
\sigma_{2}(u)=\frac{1}{M} \sigma_{1}(u) \cdot \gamma(u)
$$

$k_{1}$ and $k_{2}$ are coefficients, $u$ denotes the factor responsible for deactivation, $M$ is the number of active surface points needed for a catalytic cluster. If we denote by $\gamma(u)$ the probability that $M$ single sites are properly located to construct a catalytic cluster, we get the following relationships describing the activity changes in the course of deactivation:

$$
\begin{gathered}
\frac{\partial \varphi_{1}}{\partial u}=k_{1} \frac{\partial \sigma_{1}}{\partial u} \\
\frac{\partial \varphi_{2}}{\partial u}=k_{2} \frac{\partial \sigma_{2}}{\partial u}=k_{2} \frac{1}{M}\left\{\gamma(u) \frac{\partial \sigma_{1}}{\partial u}+\sigma_{1}(u) \frac{\partial \gamma}{\partial u}\right\}
\end{gathered}
$$

If the deactivation factor $u$ matches the fraction of surface sites blocked by coke or strongly adsorbed species, it follows from Eqs. (67) and (68) that the deactivation profiles are quite different for structure insensitive and structure sensitive reactions. The significance of the $\gamma(u)$ term should be considered. The system may get to a point of ter- 
minating the action of catalytic clusters if the density of single active sites drops to a critical level at which the probability that $M$ of them may be close enough to form a cluster approaches zero. When there are no catalyst clusters, a stepwise change in selectivity in favor of structure insensitive reaction would result.

As far as the active phase of supported heterogeneous catalysts is often non-homogeneously dispersed on the support in the form of nano-sized islands, there arises the problem of a change in the properties of active sites due to the non-uniformity of the catalyst surface. The differences in the coordination of sites arising from their location (internal or interfacial) within the island structures lead to differences in free energy, adsorption affinity, catalytic properties, and stability of action. The properties of surface atoms inside the islands are related to the coordination and particular crystallographic facets. The active sites located at the interface active phase-support are coordinately unsaturated, and because of this, they are classified as similar to the crystalline edges and corners. The model developed by Yang et al. [59] estimated the site energy (interaction energy per atom) for nine fcc metals, for the cases of 201-, 586-, 1289-atom clusters, and semi-infinite surfaces. The analysis pointed out that the free energy of the internal atoms depends on the size of the island, whereas the energy values for the lowest-coordination edge and corner sites were found to be independent of cluster size. The authors of Ref. [50] reported convincing evidence that the effect of Sn on Pt electron density depends on the particle size of the catalytic clusters as well. The differences in the site energy lead to differences in the catalytic properties of internal and interfacial active sites. There is considerable motivation for developing special models that distinguish between the properties and stability of internal and interfacial sites. Especially popular is the growing interest for elucidating the action of supported $\mathrm{Au}$ catalysts (e.g., [60-62]). Several authors have ascribed the catalyst activity of gold catalysts to charged $\mathrm{Au}^{2+}$ sites located at the boundaries with the support.

Following the understanding that metal-metal or oxide-metal interfaces can lead to the formation of sites with unique activity [63], various models have been advanced in the literature to explain catalytic behavior with account for the differences in the properties of internal and interfacial active sites. The development of models that distinguish between the properties and stability of action of internal and interfacial sites is essential for finding ways to improve the selectivity of supported bifunctional catalysts. The internal and interfacial sites may be affected in different way by the various deactivating agents. This may result in specific changes of the process selectivity.
Within the framework of the suggested approach, we shall model the average area of an active phase island by the area of a circle with efficient radius $\rho$. We shall denote the active sites located inside the active phase islands as Z-type and the interfacial active sites located along the island boundaries as Y-type sites. Let $\xi$ stand for the efficient radius of an individual surface atom of the active phase. For the fresh catalyst, the number $N_{\mathrm{Z}}^{0}$ of single sites inside the island would be proportional to the ratio $(\rho / \xi)^{2}$, whereas the number $N_{\mathrm{Y}}^{0}$ of the interfacial sites would be proportional to the magnitude of $(\rho / \xi)$ :

$$
N_{\mathrm{Z}}^{0} \propto \frac{\pi \cdot \rho^{2}}{\pi \cdot \xi^{2}}=\alpha_{\mathrm{Z}}\left(\frac{\rho}{\xi}\right)^{2}
$$

where the coefficient $\alpha_{\mathrm{Z}}$ accounts for the crystallographic characteristics of the facets.

$$
N_{\mathrm{Y}}^{0} \propto \frac{2 \pi \cdot \rho}{2 \cdot \xi}=\pi \cdot \frac{\rho}{\xi}
$$

Depending on the dispersion, the number of sites contained in the active phase entities varies within the range 10-3000. Consequently, the number $N_{\mathrm{Z}}$ of internal active sites is of similar magnitude to the number $N_{\mathrm{Y}}$ of interfacial sites. The initial ratio of these two site types on the fresh catalyst would be

$$
\frac{N_{\mathrm{Y}}^{0}}{N_{\mathrm{Z}}^{0}} \propto \frac{\pi}{\alpha_{z}} \cdot \frac{\xi}{\rho}=\alpha \cdot \xi \cdot\left(\frac{1}{\rho}\right)
$$

Considering that the average size of the active phase islands is in inverse dependence to the dispersion, we get the relationship of this ratio with the dispersion $\gamma$ of the active phase:

$$
\frac{N_{\mathrm{Y}}^{0}}{N_{\mathrm{Z}}^{0}}=\beta \cdot \xi \cdot \gamma
$$

In many bifunctional catalysts, the internal and the interfacial sites catalyze different reaction routes. In general, the routes catalyzed by various types of sites should follow different kinetic expressions, which we shall denote as $f_{\mathrm{Z}}\left(P_{j}, T\right)$ and $f_{\mathrm{Y}}\left(P_{j}, T\right)$. Assuming parallel reaction routes over $\mathrm{Z}$ - and Y-type sites and separable deactivation kinetics [58], the overall reaction rate $r_{\mathrm{ov}}$ may be presented as

$$
r_{\mathrm{ov}}=r_{\mathrm{Z}}+r_{\mathrm{Y}}=k_{\mathrm{Z}} \cdot f_{\mathrm{Z}}\left(P_{j}, T\right) \cdot a_{\mathrm{Z}}+k_{\mathrm{Y}} f_{\mathrm{Y}}\left(P_{j}, T\right) \cdot a_{\mathrm{Y}}
$$

where $r_{\mathrm{Z}}$ and $r_{\mathrm{Y}}$ stand for the rates of routes facilitated by the internal and interfacial sites, $f_{\mathrm{Z}}\left(P_{j}, T\right)$ and $f_{\mathrm{Y}}\left(P_{j}, T\right)$ for their kinetic expressions. The $a_{\mathrm{Z}}$ and $a_{\mathrm{Y}}$ functions stand for the activity of the site types, and are linearly dependent on their number

$$
a_{\mathrm{Z}} \propto N_{\mathrm{Z}} / N_{\mathrm{Z}}^{0} \text {, and } a_{\mathrm{Y}} \propto N_{\mathrm{Y}} / N_{\mathrm{Y}}^{0}
$$

For a fresh catalyst, we get the following dependencies of the initial selectivity on dispersion: 


$$
\begin{aligned}
S_{\mathrm{Z}}^{0}= & \frac{r_{\mathrm{Z}}^{0}}{r_{\mathrm{Z}}^{0}+r_{\mathrm{Y}}^{0}}=\frac{k_{\mathrm{Z}} f_{\mathrm{Z}}\left(P_{j}, T\right) \cdot N_{\mathrm{Z}}^{0}}{k_{\mathrm{Z}} f_{\mathrm{Z}}\left(P_{j}, T\right) \cdot N_{\mathrm{Z}}^{0}+k_{\mathrm{Y}} f_{\mathrm{Y}}\left(P_{j}, T\right) \cdot N_{\mathrm{Y}}^{0}} \\
& \frac{1}{1+\frac{k_{\mathrm{Y}} f_{\mathrm{Y}}\left(P_{j}, T\right)}{k_{\mathrm{Z}} f_{\mathrm{Z}}\left(P_{j}, T\right)} \cdot \frac{N_{\mathrm{Y}}^{0}}{N_{\mathrm{Z}}^{0}}}=\frac{1}{1+k_{\mathrm{s}} \xi \frac{f_{\mathrm{Y}}\left(P_{j}, T\right)}{f_{\mathrm{Z}}\left(P_{j}, T\right)} \cdot \gamma}
\end{aligned}
$$

and

$$
\begin{gathered}
S_{\mathrm{Y}}^{0}=\frac{r_{\mathrm{Y}}^{0}}{r_{\mathrm{Z}}^{0}+r_{\mathrm{Y}}^{0}}=\frac{k_{\mathrm{Y}} f_{\mathrm{Y}}\left(P_{j}, T\right) \cdot N_{\mathrm{Y}}^{0}}{k_{\mathrm{Z}} f_{\mathrm{Z} 1}\left(P_{j}, T\right) \cdot N_{\mathrm{Z}}^{0}+k_{\mathrm{Y}} f_{\mathrm{Y}}\left(P_{j}, T\right) \cdot N_{\mathrm{Y}}^{0}}= \\
\frac{1}{1+\frac{k_{\mathrm{Z}} f_{\mathrm{Z}}\left(P_{j}, T\right)}{k_{\mathrm{Y}} f_{\mathrm{Y}}\left(P_{j}, T\right)} \cdot \frac{N_{\mathrm{Z}}^{0}}{N_{\mathrm{Y}}^{0}}}=\frac{1}{1+\frac{1}{k_{\mathrm{s}} \xi} \cdot \frac{f_{\mathrm{Z}}\left(P_{j}, T\right)}{f_{\mathrm{Y}}\left(P_{j}, T\right)} \cdot \frac{1}{\gamma}}
\end{gathered}
$$

for $k_{\mathrm{s}}=\frac{k_{\mathrm{Y}}}{k_{\mathrm{Z}}} \beta \xi$.

Considering that the intrinsic kinetic laws $f_{\mathrm{Z}}\left(P_{j}, T\right)$ and $f_{\mathrm{Y}}\left(P_{j}, T\right)$ are functions of temperature and partial pressures, but not of dispersion, it follows from Eqs. (75) and (76) that a high dispersion of the active phase on the fresh catalyst favors the reactions facilitated by interfacial active sites.

It is reasonable to expect different vulnerability of the internal and interfacial active sites under the action of various deactivation factors. If the process is accompanied by coke formation, the deposition of a carbon layer on the catalyst surface hinders the access of the reactants to the active sites. This can be interpreted as the decrease of the average size of the active phase islands (or the efficient radius $\rho$ ). Considering equations (69) and (70), we get the following expressions for the variation of the activity functions for internal $\left(a_{\mathrm{Z}}\right)$ and interfacial $\left(a_{\mathrm{Y}}\right)$ sites:

$$
\begin{aligned}
\mathrm{d} a_{\mathrm{Z}} \propto \mathrm{d} N_{\mathrm{Z}} & =\kappa_{\mathrm{Z}}\left(\frac{2}{\xi^{2}} \rho\right) \mathrm{d} \rho=\kappa_{\mathrm{Z}}\left(\frac{2}{\xi}\right)\left(\frac{\rho}{\xi}\right) \mathrm{d} \rho \\
\mathrm{d} a_{\mathrm{Y}} & \propto \mathrm{d} N_{\mathrm{Y}}=\kappa_{y}\left(\frac{\pi}{\xi}\right) \mathrm{d} \rho
\end{aligned}
$$

As can be seen, coke formation caused the decrease of the island sizes, which will affect in different ways and degrees the activity of the internal and the interfacial sites. The decrease of the interfacial-type activity will follow the decrease of the efficient size of the active phase entities. The effect will be more pronounced for a larger size $(\xi)$ of the individual metal atoms constituting the active phase. The activity of the internal sites is expected to be different because of the dependence of $d a_{\mathrm{Z}}$ on the $\rho / \xi$ ratio.

As the efficient island size $\rho$ will be reduced in the course of coke formation, the activity of the Z-type sites will be most affected by the earlier coke deposits. The deterioration effect would slow down as the entities of the active phase decrease in size.

It can be estimated on comparing Eqs. (77) and (78) that

$$
\mathrm{d} a_{\mathrm{Z}}=\left(\frac{2}{\pi}\right)\left(\frac{\rho}{\xi}\right) \mathrm{d} a_{\mathrm{Y}}
$$

As the efficient size of the active phase islands $(\rho)$ is one to three orders of magnitude larger than the sizes of the constituent metal atoms, it is reasonable to assume $\rho / \xi$ $>>1$. Consequently, $\mathrm{d} a_{\mathrm{Z}}>>\mathrm{d} a_{\mathrm{Y}}$, that is, the internal active sites will be more severely affected by the deactivation event.

The relevant trend of selectivity variation can be derived as follows. On substituting $k_{\mathrm{s}} \gamma=k_{\rho} / \rho$ in Eqs. (75) and (76), we get the dependencies of selectivity on the current size of the active phase nano-structures:

$$
S_{\mathrm{Z}}=\frac{1}{1+k_{\rho} \frac{f_{\mathrm{Y}}\left(P_{j}, T\right)}{f_{\mathrm{Z}}\left(P_{j}, T\right)} \cdot \frac{\xi}{\rho}}
$$

and

$$
S_{\mathrm{Y}}=\frac{1}{1+\frac{1}{k_{\rho}} \frac{f_{\mathrm{Z}}\left(P_{j}, T\right)}{f_{\mathrm{Y}}\left(P_{j}, T\right)} \cdot \frac{\rho}{\xi}}
$$

Differentiating these relations, one obtains the corresponding trends for the selectivity changes:

$$
\mathrm{d} S_{\mathrm{Z}}=\frac{k_{\mathrm{s}} \frac{f_{\mathrm{Y}}\left(P_{j}, T\right)}{f_{\mathrm{Z}}\left(P_{j}, T\right)}}{\xi\left(k_{\mathrm{s}} \frac{f_{\mathrm{Y}}\left(P_{j}, T\right)}{f_{\mathrm{Z}}\left(P_{j}, T\right)}+\frac{\rho}{\xi}\right)^{2}} \cdot \mathrm{d} \rho
$$

It follows from this relationship that the change of selectivity towards the reactions facilitated by internal active sites will change synchronously with the change of the effective size $\rho$ of the active phase islands. As coking results in the reduction of $\rho$, the selectivity for such routes will decrease in the course of deactivation. The drop will be more pronounced at the start of the deactivation process.

$$
\mathrm{d} S_{\mathrm{Y}}=(-)\left\{\frac{k_{\mathrm{s}} \frac{f_{\mathrm{Y}}\left(P_{j}, T\right)}{f_{\mathrm{Z}}\left(P_{j}, T\right)}}{\xi\left(k_{\mathrm{s}} \frac{f_{\mathrm{Y}}\left(P_{j}, T\right)}{f_{\mathrm{Z}}\left(P_{j}, T\right)}+\frac{\rho}{\xi}\right)^{2}}\right\} \cdot \mathrm{d} \rho
$$

By contrast, the selectivity towards reactions catalyzed by interfacial active sites will increase with the decrease of the effective size of the active phase island in the course of coke covering.

\section{Catalytic processes on intentionally poisoned catalysts}

The partial oxidation of ethylene to ethylene oxide (EO) over silver catalysts is always accompanied by the highly exothermic complete oxidation of ethylene: 
(I) $\mathrm{C}_{2} \mathrm{H}_{2}+1 / 2 \mathrm{O}_{2}=\mathrm{C}_{2} \mathrm{H}_{4} \mathrm{O}+28 \mathrm{kcal} / \mathrm{mol}$

(II) $\mathrm{C}_{2} \mathrm{H}_{4}+3 \mathrm{O}_{2}=2 \mathrm{CO}_{2}+2 \mathrm{H}_{2} \mathrm{O}+316 \mathrm{kcal} / \mathrm{mol}$

The second reaction is undesired as it results in the loss of expensive ethylene and it hampers the maintenance of the optimum catalyst bed temperature. Suppression of the total oxidation reaction, i.e., increased selectivity for EO, is achieved in industry by adding a gas phase selectivity promoter, usually 1,2-dichloroethane (DCE) in the range of 1 to 28 ppm.

The kinetic models for selective and total oxidation of ethylene in the presence of DCE [15-19] are given below. It should be noted that the partial pressure of DCE participates in the rate equations:

$$
\begin{array}{r}
r(\mathrm{I})=\frac{k_{1} P_{\mathrm{O}_{2}} P_{\mathrm{E}}-k_{2} P_{\mathrm{O}_{2}} P_{\mathrm{E}} P_{\mathrm{DCE}}^{0.19}}{1+k_{5} P_{\mathrm{O}_{2}}+k_{6} P_{\mathrm{E}}} \\
r(\mathrm{II})=\frac{k_{3} P_{\mathrm{O}_{2}} P_{\mathrm{E}}-k_{4} P_{\mathrm{O}_{2}} P_{\mathrm{E}} P_{\mathrm{DCE}}^{0.07}}{1+k_{5} P_{\mathrm{O}_{2}}+k_{6} P_{\mathrm{E}}}
\end{array}
$$

The selectivity of the process toward EO is given by

$$
S_{\mathrm{EO}}=\frac{6\left(k_{1}-k_{2} P_{\mathrm{DCE}}^{0.19}\right)}{\left(5+\frac{F_{\mathrm{E}}}{F_{\mathrm{O}_{2}}}\right)\left(k_{1}-k_{2} P_{\mathrm{DCE}}^{0.19}\right)+\frac{6 F_{\mathrm{E}}}{F_{\mathrm{O}_{2}}}\left(k_{3}-k_{4} P_{\mathrm{DCE}}^{0.07}\right)}
$$

where $F_{\mathrm{E}}$ and $F_{\mathrm{O} 2}$ are the ethylene and the oxygen flow rates. This expression describes the control of the selectivity by changing the input parameters like ethylene and oxygen flow rates and the partial pressure of DHE. The average deviation for the experimental selectivity from the selectivity given by Eq. (86) is $\pm 6 \%$.

The most important factor influencing the selectivity was the partial pressure of the promoter DCE in the feed gas stream. This dependence may be expressed by

$$
S=a C_{\mathrm{DCE}}+b
$$

This is a straight line equation. The slope " $a$ " and the intercept " $b$ " depend on the reaction temperature and feed composition. The physical meaning of " $b$ " is the selectivity in the absence of DCE and " $a$ " is the sensitivity of the selectivity to DCE concentration. The sensitivity of the selectivity is highest at the highest reaction temperatures.

The deactivation of silver catalysts promoted by chlorinated compounds is reversible. The activity " $a$ " of the pellet at any time is defined as the ratio of the rate, at which the pellet converts the ethylene at given moment, and the rate of the reaction of ethylene on a fresh pellet. The rate at which the activity function " $a$ " decreases with time " $t$ " is then expressed by:

$$
\frac{\mathrm{d} a}{\mathrm{~d} t}=k_{\mathrm{d}} C_{\mathrm{p}}^{n}
$$

where $C_{\mathrm{p}}$ is the poison concentration, $k_{\mathrm{d}}$ is the rate constant for the deactivation and " $n$ " is the order of the deactivation reaction.
At steady-state conditions, the activity of the catalysts does not change with time and $\mathrm{d} a / \mathrm{d} t=0$ and $a=$ const.

In this case the activity functions " $a_{1}$ " and " $a_{2}$ " for both selective and total oxidation are functions only of the promoter partial pressure in the feed:

$$
\begin{aligned}
& a_{1}=1-\frac{k_{2}}{k_{1}} P_{\mathrm{DCE}}^{0.19} \\
& a_{2}=1-\frac{k_{4}}{k_{3}} P_{\mathrm{DCE}}^{0.07}
\end{aligned}
$$

It is obvious that the degree of deactivation will be bigger when the promoter concentration is higher. The model predicted that at certain DCE concentrations (but at different concentrations of DCE for selective and total oxidation), the catalyst will be completely poisoned. This was observed experimentally at concentrations of DCE $120 \mathrm{ppm}$ for selective oxidation and $40 \mathrm{ppm}$ for total oxidation. Therefore, under certain conditions, the reaction proceeds with $100 \%$ selectivity for EO, but at a very low conversion, which is of insignificant industrial importance.

In the industrial process of the selective catalytic oxidation of ethene, the feed contains a high concentration of carbon dioxide up to $25 \%$. It was found that the $\mathrm{CO}_{2}$ obtained from the total ethylene oxidation in concentrations up to $10 \%$ has no effect on the process, and can be regarded as inactive ballast gas [64]. At concentrations above $10 \%, \mathrm{CO}_{2}$ has a retardation effect on both selective and full oxidation, i.e., it is also a selectivity promotor arising from the reaction itself. A kinetic model was developed that involved two rate equations, reflecting the carbon dioxide inhibiting effect on the rate of epoxidation $r(\mathrm{I})$ and the rate of total oxidation of ethene $r$ (II), by including the carbon dioxide partial pressure in their functional form.

$$
\begin{array}{r}
r(\mathrm{I})=\frac{k_{1} P_{\mathrm{O}_{2}} P_{\mathrm{E}}-k_{2} P_{\mathrm{O}_{2}} P_{\mathrm{E}} P_{\mathrm{CO}_{2}}}{1+k_{5} P_{\mathrm{O}_{2}}+k_{6} P_{\mathrm{E}}+k_{7} P_{\mathrm{CO}_{2}}} \\
r(\mathrm{II})=\frac{k_{3} P_{\mathrm{O}_{2}} P_{\mathrm{E}}-k_{4} P_{\mathrm{O}_{2}} P_{\mathrm{E}} P_{\mathrm{CO}_{2}}}{1+k_{5} P_{\mathrm{O}_{2}}+k_{6} P_{\mathrm{E}}+k_{7} P_{\mathrm{CO}_{2}}}
\end{array}
$$

The models are valid for the temperature range 240-292 ${ }^{\circ} \mathrm{C}$, and carbon dioxide concentrations in the range $12 \%-33 \%$. The experimental data and model predictions have shown that the retardation effect of carbon dioxide is stronger for the reaction of full oxidation than selective oxidation. Therefore, carbon dioxide has a favourable effect on the selectivity for EO production, and is an additional instrument for selectivity control.

The kinetic model presented by Eqs. (84)-(86) was successfully used for modeling an industrial ethylene oxide plant [65].

\section{Accelerated method for catalyst lifetime estimation}


The most time consuming step in the design and development of industrial catalysts and in quality checking procedures is the estimate of catalyst lifetime. An express method for a one-day laboratory test was proposed for the prognosis of the activities and lifetimes of catalysts for several industrial processes: gas phase hydrogenation of nitrobenzene to aniline, gas phase crotonaldehyde hydrogenation to butanol, liquid phase hydrogenation of crotonaldehyde to butiraldehyde, gasoline sweetening process in trickle bed reactor [38,66-68].

The method is based on the knowledge of the deactivation behaviour of the catalysts under different reaction conditions, including extreme conditions of very high liquid hourly space velocity (LHSV), and it can predict the industrial lifetime and performance using data obtained from a one-day test under laboratory conditions.

It is well known that different LHSV values determine different lifetimes of the catalyst. Figure 7 presents the life time/LHSV dependence for different aniline production catalysts. The LHSV/lifetime curve is the property of a given catalyst. It has reproducible shape characteristics for any single catalyst, which have to be established experimentally. Any point from the LHSV/lifetime curve can be used for lifetime estimation.

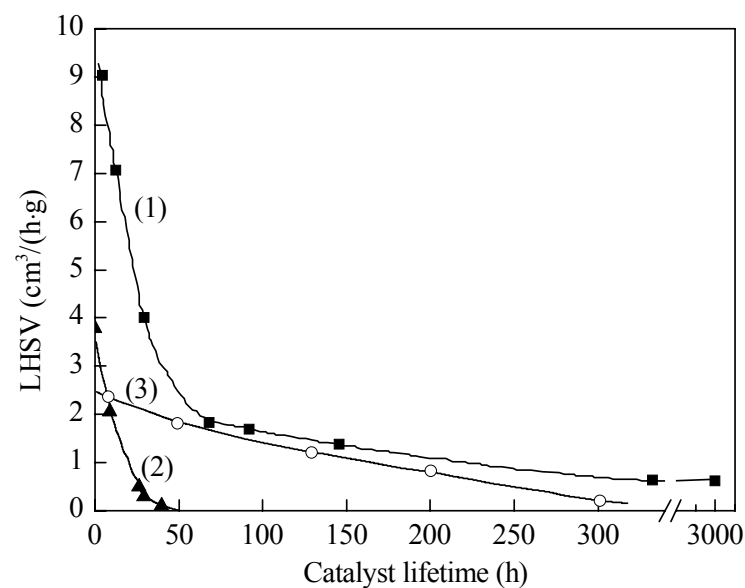

Fig. 7. LHSV/catalyst lifetime dependence for a copper catalyst for gas phase hydrogenation of nitrobenzene to aniline [68].

It is clear that the aim of the express lifetime estimation is to obtain reliable result in a possibly shortest period of time. Therefore, it is expedient to use very high LHSV. At these conditions, serious problems with heat and mass transfer may appear.

The LHSV/lifetime curve for a process of gas phase hydrogenation of nitrobenzene to aniline on kieselguhr-supported copper catalyst was prepared using data collected from different reactors: (i) Laboratory glass reactor containing $1 \mathrm{~g}$ catalyst with particle size $0.1 \mathrm{~mm}$, (ii) Hungarian made pilot system OL-105/01 containing $5 \mathrm{~g}$ catalyst with particle size $1 \mathrm{~mm}$, (iii) Pilot plant reactor containing $2 \mathrm{~kg}$ industrial pellets with size $6 \times 6 \mathrm{~mm}$, (iv) Pilot plant reactor containing $20 \mathrm{~kg}$ industrial pellets with size $6 \times 6 \mathrm{~mm}$, (v) Industrial unit for aniline production with four packed bed reactors arranged in series (each reactor contained $4000 \mathrm{~kg}$ catalyst with pellet size $6 \times 6 \mathrm{~mm}$ ). All experimental points fell well along the same curve.

As an illustration, the curves in Fig.7 present the data used for estimation of the lifetime of three different catalysts (namely: Sample 1 - a Cu catalyst; Sample 2 - a Ni catalyst; Sample 3 - a Ni-Cu catalyst) efficient for gas phase nitrobenzene to aniline hydrogenation. At industrial conditions of nitrobenzene LHSV $=0.5 \mathrm{~cm}^{3} /(\mathrm{h} \cdot \mathrm{g})$ and temperature of $170{ }^{\circ} \mathrm{C}$ and $\mathrm{H}_{2}$ :nitrobenzene molal ratio $1: 15$, the lifetime of the catalyst for aniline production was about 3000 h (curve 1 in Fig. 7). If we perform the catalytic activity test at a much higher nitrobenzene LHSV i.e. 8 $\mathrm{cm}^{3} /(\mathrm{h} \cdot \mathrm{g})$, the catalyst lifetime will be only $7 \mathrm{~h}$. In this way, the one-day test can predict the catalyst lifetime.

The deactivation curves characterizing Sample 2 and Sample 3 correspond to curves 2 and 3 in Fig. 7. These catalysts were less stable, deactivate faster than Sample 1 and their lifetime were much shorter than the lifetime of Sample 1.

The efficiency of the proposed method was tested and elaborated on in different industrial units for catalyst production and for catalyst use.

\section{Concluding remarks}

In deriving kinetic models for processes accompanied by catalyst deactivation, particular attention should be paid to correlating the kinetics of the main reaction and the deactivation kinetics with the reaction mechanism. Knowing the relationship between the mechanism and catalyst deactivation, one can predict more successfully the behavior of the reaction system under different operation conditions. Special attention should be paid to the selectivity changes in the course of catalyst deactivation.

Catalyst deactivation has different kinetics depending on the mechanism of blockage of active sites. In many processes, the formation of the blocking agents is a constituent part of the reaction mechanism. The deactivation kinetics is linked to the peculiarities of the generation of the deactivating agents.

Various mechanisms were considered for the formation of coke precursors for the feasible interactions of different surface intermediates with either initial reactants or products from the gas phase. Assuming that the formation of coke precursors is the limiting step of the deactivation reaction, the deactivation kinetics should be considered as functions of the surface concentrations of the intermediates and the 
partial pressures of the gas phase reactants responsible for deactivation.

When modeling deactivation kinetics of processes affected by intraparticle diffusion, account was taken of the changes in the reaction rate caused by mass transfer limitation inside the catalyst pores and also the modifications of the rate of deactivation caused by diffusion resistance. The analysis indicated that for a number of processes, the decrease of the reaction rate due to deactivation is weaker under diffusion control. The superposition of the opposing effects may bring about mutual compensation. For certain reaction mechanisms, there exists a domain of operation conditions where the effective rate of the process under diffusion control may even exceed the rate in the kinetic region.

A particular approach was used to model the distribution of coke deposits along the radius of the catalyst particle, which depends on the mechanism of their generation. Calculations that considered the changes in selectivity of processes accompanied by catalyst deactivation indicated that under the action of deactivation, the selectivity may either decrease or increase depending on the reaction mechanism. The changes were larger in the absence of intraparticle diffusion.

An approach was suggested for distinguishing different types of active sites responsible for different routes on bifunctional catalysts. The model assumed non-uniform vulnerability of active sites, depending on their location. Problems concerning the relationship of the dispersion of the active phase with process selectivity were discussed. The effect of coke formation on the contribution of different site types to the evolution of activity was analyzed.

The kinetic models may have practical use elucidating ways for reducing the harmful effect of deactivation on the yield of target products.

\section{References}

1 Hughes R. Deactivation of Catalysts. New York: Academic Press, 1984

2 Bartholomew C H. Appl Catal A, 2001, 212: 17

3 Oudar J, Wise H. Deactivation and Poisoning of Catalysts. New York: Marcel Dekker, 1985

4 Butt J B, Petersen E E. Activation, Deactivation and Poisoning of Catalysts. San Diego: Academic Press, 1988

5 Al-Dalama K, Stanislaus A. Chem Eng J, 2006, 120: 33

6 Hegedus L I, McCabe R W. Catalyst Poisoning. New York: Marcel Dekker, 1984

7 Krishna A S. Catal Rev-Sci Eng, 1990, 32: 279

8 Duprez D. Appl Catal A, 1992, 82: 111

9 Farrauto B R, Bartholomew C H. Fundamentals of Industrial Catalytic Processes. London: Chapman \& Hall, Kluwer Academic Publishers, 1997
10 Twig M V, Spencer M S. Appl Catal A, 2001, 212:161

11 Thomas J M, Thomas W J. Principles and Practice of Heterogeneous Catalysis. Weinheim: VCH, 2005

12 Furimsky E, Massoth F E. Catal Today, 1999, 52: 381

13 Froment G F. Appl Catal A, 2001, 212: 117

14 Murzin D Y, Avetisov A K. Chem Eng Sci, 1998, 53: 2469

15 Petrov L, Eliyas A, Shopov D. Appl Catal, 1985, 18: 87

16 Petrov L, Eliyas A, Shopov D. Appl Catal, 1986, 24: 145

17 Eliyas A, Petrov L, Shopov D. Appl Catal, 1988, 41: 39

18 Petrov L, Eliyas A. Izv Khim, 1988, 21: 289

19 Liu W, Eliyas A, Petrov L. Appl Catal, 1990, 61: 265

20 Wolf E E, Petersen E E. J Catal, 1977, 46: 190

21 Wolf E E, Petersen E E. J Catal, 1977, 47: 28

22 Levenspiel O. J Catal, 1972, 25: 265

23 Froment G F. Stud Surf Sci Catal, 1980, 6: 1

24 Butt J B, Wachter C K, Billimoria R M. Chem Eng Sci, 1978, 33: 1321

25 Kumbilieva K, Kiperman S L, Petrov L. Collect Czech Chem Commun, 1992, 57: 2452

26 Kumbilieva K, Kiperman S L, Petrov L. Kinet Katal, 1995, 36: 73

27 Wheeler A. Adv Catal, 1955, 2: 105

28 Satterfield C N. Mass Transfer in Heterogeneous Catalysis. Cambridge: Academic Press, 1970

29 Froment G F, Bischoff K B. Chemical Reactor Analysis and Design. New York: Willey, 1990

30 Yu L C, Butt J B. Stud Surf Sci Catal, 1991, 68: 343

31 Temkin M I. Adv Catal, 1979, 28: 173

32 Best D A, Wojciechowski B W. Can J Chem Eng, 1976, 54: 197

33 Zhorov Y M. Kinetics of Industrial Organic Reactions. Moscow: Nauka, 1989

34 Kumbilieva K, Kostyukovsky M M, Petrov L, Kiperman S L. Stud Surf Sci Catal, 1987, 34: 381

35 Kumbilieva K, Kostyukovsky M M, Petrov L, Kiperman S L. Chem Eng Sci, 1988, 43: 1195

36 Kumbilieva K, Sergeeva T Y, Lok L K, Petrov L, Kiperman S L. Appl Catal A, 1992, 82: 159

37 Pshezhetskii S, Rubinstein R. Zh Fiz Khim, 1946, 20: 207

38 Petrov L, Kumbilieva K, Kirkov N. Appl Catal, 1990, 59: 31

39 Gaidai N A, Butovsky M E, Kol'tsov H I, Kiperman S L, Kotel'nikov G R. Kinet Katal, 1977, 18: 1463

40 Kumbilieva K, Petrov L, Kiperman S L. Stud Surf Sci Catal, 1994, 88: 175

41 Kumbilieva K, Petrov L, Kiperman S L. Appl Catal A, 1994 118: 199

42 Kiperman S L. Kinet Katal, 1981, 22: 1463

43 Yang S H, Satterfield C N. J Catal, 1983, 81: 168

44 Ozkan U S, Zhang L, Ni Sh, Moctezuma E. J Catal, 1994, 148: 181

45 Topsǿe H, Clausen B S, Massoth F E. Hydrotreating Catalysis. Berlin: Springer-Verlag, 1996

46 Sachtler W H M. J Mol Catal, 1984, 25: 1

47 Biloen P, Dautzenberg F M., Sachtler W M H. J Catal, 1977, 50: 77 
48 Cortright R D, Dumesic J A. J Catal, 1994, 148: 771

49 Meriaudeau P, Naccache C, Thangaraj A, Bianchi C L, Carli R, Vishvanathan V, Narayanan S. J Catal, 1995, 154: 345

50 Merlen E, Beccat P, Bertollini J C, Delichere P, Zanier N, Didillon B. J Catal, 1996, 159: 178

51 Beeckman J W, Froment G F. Ind Eng Chem Fundam, 1979, 18: 245

52 Beeckman J W, Froment G F. Ind Eng Chem Fundam, 1982, 21: 243

53 Kumbilieva K, Petrov L. Chin J Catal, 2010, 31: 1

54 Kiperman S L, Kumbilieva K, Petrov L. Ind Eng Chem Res, 1989, 28: 376

55 Lok L K, Gaidai N A, Kiperman S L. Kinet Katal, 1986, 27: 1112

56 Lok L K, Gaidai N A, Kiperman S L, Kogan S B. Kinet Katal, 1990, 31: 483

57 Kumbilieva K, Gaidai N A, Nekrasov N V, Petrov L, Lapidus A L. Chem Eng J, 2006, 120: 25

58 Kogan S B, Podkletnova N M, Ilyasova A S, Bursian N R.
Zhurn Prikl Khimii, 1983, 1832

59 Yang L Q, De Pristo A E. J Catal, 1994, 148: 575

60 Haruta M, Tsobota S, Kobayashi T, Kageyana H, Genet M G, Delmon B. J Catal, 1993, 144: 175

61 Bond G C, Thompson D T. Catal Rev-Sci Eng, 1999, 41: 319

62 Petrov L. Stud Surf Sci Catal, 2000, 130: 2345

63 Somorjai G A. Catal Today, 1992, 12: 343

64 Eliyas A, Petrov L. Appl Catal, 1990, 62: 11

65 Cornelio A A. Indian Chem Eng, Sect A, 2006, 48: 164

66 Petrov L, Vladov Ch, Neshev N, Bonev Ch, Prahov L, Kirkov N, Vasileva M, Filkova D, Dancheva S. BG 41960. 1986

67 Petrov L, Vladov Ch, Bonev Ch, Prahov L, Kirkov N, Eliyas A, Neshev N, Filkova D, Dancheva S. In: EuropaCat-2, Book of Abstracts, Maastricht, The Netherlands, 1995. 646

68 Petrov L. In: Derouane E G, Parmon V N, Lemos F, Ribeiro F R eds. Principles and Methods for Accelerated Catalyst Design and Testing. NATO Science Series II: Mathematics, Physics and Chemistry, Vol. 19. New York: Kluwer Academic Publishers, 2002. 69 